Article (Special Issue "New Materials and Techniques for Orthodontics")

\title{
3D printed orthodontic distalizer with individual base for tooth- borne hybrid approach in class II unilateral malocclusions treat- ment
}

Andrej Thurzo ${ }^{1 *}$, Wanda Urbanová ${ }^{2}$, Bohuslav Novák ${ }^{1}$, Iveta Waczulíková ${ }^{3}$ and Ivan Varga ${ }^{4}$,

1 Department of Stomatology and Maxillofacial Surgery, Faculty of Medicine, Comenius University in Bratislava, 81250 Bratislava, Slovakia; bohuslav.novak@fmed.uniba.sk

2 Department of Orthodontics and Cleft Anomalies, Dental Clinic 3rd Medical Faculty Charles University, Faculty Hospital Kralovske Vinohrady, Prague, Czech Republic; wanda.urbanova@gmail.com

3 Department of Nuclear Physics and Biophysics, Faculty of Mathematics, Physics and Informatics, Comenius University, Mlynská dolina F1, 84248 Bratislava, Slovakia; iveta.waczulikova@fmph.uniba.sk

4 Institute of Histology and Embryology, Faculty of Medicine, Comenius University in Bratislava, 81372 Bratislava, Slovakia; ivan.varga@fmed.uniba.sk

* Correspondence: Andrej.Thurzo@fmed.uniba.sk ; Tel.: (+421 903110 107)

\begin{abstract}
Aim of this research paper is to introduce a novel method of hybrid orthodontic toothborne distalizer treatment of class II malocclusion by using 3D printed biocompatible personalized distalizer. Explains 3D designing, printing and clinical application of individualized biocompatible medical device dedicated for orthodontic teeth distalization. Compares such distalizer manufactured from two different biocompatible photopolymers (white and transparent). Evaluates their clinical performance and also patients' aesthetical perception. Clinical part includes comparison of treatment debonding on the set of 12 complete orthodontic treatments with unilateral class II malocclusion managed with hybrid approach (CAT-Invisalign with 3D printed distalizer). Paper offers an evaluation of the personalized distalizer functioning in regard to current publications and comparison to conventional prefabricated alternatives like Carriere ${ }^{\circledR}$ Distalizer ${ }^{\mathrm{TM}}$ appliance. Results showed no significance of material differences on clinical performance of such individualized distalizers. Research showed preference of patients towards transparent biocompatible photopolymer instead of white A2 shade.
\end{abstract}

Keywords: biocompatible 3D printing, photopolymers, orthodontics, distalizer, CAD/CAM, personalized treatment, computer modelling;

\section{Abbreviations}

Table 1. Table of used abbreviations in alphabetical order.

\begin{tabular}{cl}
\hline Abbreviation & \multicolumn{1}{c}{ Meaning } \\
\hline 3D & Three-dimensional \\
AM & Additive Manufacturing \\
CAT & Clear Aligner Therapy \\
CDA & Carriere Distalizer Appliance \\
CMA & Carriere Motion 3D Appliance ${ }^{1}$ \\
STL & Standard Tessellation Language, describes only the surface geometry of a \\
& three-dimensional object without any representation of color or texture \\
\hline
\end{tabular}




\section{Introduction}

Recent progress in the field of digitalization enabled by digital impressions and the digital workflow in general, opened many opportunities in orthodontic devices customization. Advance of the material research of dental composites, including the dental composite resins intended for 3D printing, brought a rapid advance in more personalized orthodontics. Research led to significant improvements of mechanical strength, surface characteristics and biocompatibility of composites used in dentistry [1 - 8]. Focus on new bioactive compounds in composites to prevent dental caries development and progression has led researchers to use of nanotechnologies [9, 10]. Composite configuration has significant impact not only on 3D-printed appliance properties [11 - 13] but also on adhesion efficacy when used as adhesive for an appliance [14, 15].

Prevalence of malocclusion and orthodontic treatment need is well explored. Noticeable incisor irregularity occurs in the majority of all racial/ethnic groups, with only $35 \%$ of adults having well-aligned mandibular incisors [16]. Irregularity is severe enough in $15 \%$ where both social acceptability and function could be affected, and major arch expansion or extraction of some teeth would be required for correction. About $20 \%$ of the population have deviations from the ideal bite relationship; in $2 \%$ these are severe enough to be disfiguring and are at the limit for orthodontic correction. Application of the Index of Treatment Need reveals that $57 \%$ to $59 \%$ of each racial/ethnic group has at least some degree of orthodontic treatment need [16].

This article introduces a novel method in personalized 3D designing and printing of biocompatible distalizer from two different biocompatible photopolymers. This 3Dprinted customized distalizer could be categorized with Carriere Motion 3D Appliances (CMA) as the biomechanical principles are very similar. Especially its applications, in combination with Clear Aligner Therapy (CAT).

Both clear aligners and braces are effective in treating malocclusion. Clear aligners had an advantage in segmented movement of teeth and shortened treatment duration, but were not as effective as braces in producing adequate occlusal contacts, controlling teeth torque, and retention [17]. CAT in general is considered efficient orthodontic treatment. It aligns and levels the arches; and is effective in controlling anterior intrusion but not anterior extrusion. It is effective in controlling posterior buccolingual inclination but not anterior buccolingual inclination. It is effective in controlling upper molar bodily movements; and is less effective in controlling rotation of rounded teeth in particular [18].

Various techniques have been presented for orthodontic therapy of class II malocclusions. In some non-extraction protocols, maxillary molar distalization can be applied to correct molar relationships in orthodontic patients with maxillary dentoalveolar protrusion $[19,20]$.

To prevent un-necessary extraction of mostly upper premolars, the upper molars can be distalized by means of extraoral or intraoral forces [21]. Recently, several techniques have been presented to reduce the dependence on patient compliance, such as intraoral appliances with and without skeletal anchorage. However, even these devices produce undesirable tipping of the maxillary molars as well as loss of anterior anchorage to some extend during distalization movement [22 - 23].

An extensive comprehensive literature research regarding treatment effects of Carriere Motion Appliance (CMA) on class II patients was performed. This resulted in seventeen studies, albeit with absence of valuable randomized controlled trials. All of these publications evaluated the skeletal changes as not significant. With respect to the dentoalveolar changes, an increased lower incisor's proclination (L1-MP) was observed. CMA also caused an increase in the airway volume, an increase in the masseter and temporalis muscles activities and a minor relapse of malocclusion after 4-years of follow-up. The results should be taken with caution because only a secondary level of evidence was found. The CMA used for the treatment of class II malocclusion did not cause skeletal changes; however, mostly dento-alveolar effects were noticed [19, 23]. In the last decades, increasing numbers of adult patients have pursued orthodontic treatment and expressed a desire 
for aesthetic and comfortable alternatives to conventional fixed appliances. Clear aligner therapy (CAT) was introduced as a reaction to this demand. The patient compliance is the prime weakness of CAT. Patients nowadays frequently prefer aesthetical variants of the treatment over more effective ones. They gather information about treatment possibilities not only from official web-sites but also from social networks of their peers, idols or preferred clinics or clinicians [19, 24].

In biomechanically demanding orthodontic clinical situations, where patient compliance is crucial, a fixed orthodontic appliance might be more suitable than a removable one. Teeth distalization is such a difficult teeth movement. 3D printed "CMA-like" distalizer concept intended as tooth-borne fixed appliance bonded with adhesive on tooth enamel surface is introduced in this article. In wider context it is meant for hybrid class II treatment with CAT (Figure $1 \mathbf{a}, \mathbf{b})$.

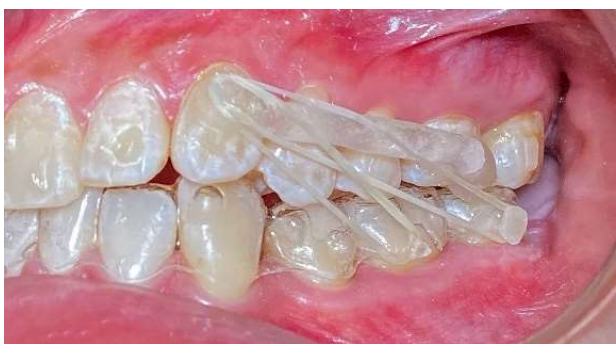

(a)

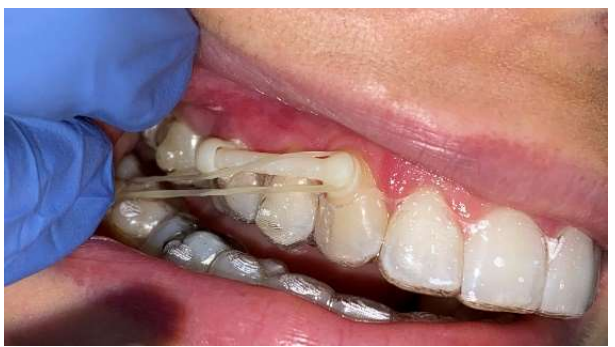

(b)

Figure 1. Examples of 3D-printed distalizers with individual base for tooth-borne hybrid approach in class II unilateral malocclusions treatment, comparison of two biocompatible resins (a) transparent resin- Dental LT Clear V2 from Formlabs; (b) Opaque Denture Teeth resin from Formlabs.

In research by Rossini et al. it has been concluded that maxillary molar distalization up to $2.5 \mathrm{~mm}$ is one of the most predictable movements with CAT $[19,25,26]$. This significant predictability was achieved through combination of staging, the use of proper attachment configuration [19, 26], and full-day class II elastics (with parameters of 0.25 in, $4.5 \mathrm{oz})$.

As stated above the CAT that includes difficult tooth movements like significant distalization, is even more sensitive to patient compliance. The CAT success strongly depends on the cooperation of the patient [27].

Class II treatments with CAT require mean treatment times of approximately 20 months during which class II elastics need to be used all day from treatment beginning until class I canine relationship has been achieved [19, 28].

Curative devices (like distalizers) should be comfortable for the patient, provide rapid and effective treatment, and favor patient compliance with such hybrid orthodontic treatment (that includes aesthetics aspect) [19]. That is one of the reasons why this article compares two different biocompatible materials. A clear (transparent) composite resin and an opaque white (tooth color) resin. Clear aligners are comfortable and aesthetically acceptable as already discussed $[29,30]$, and require strong patient compliance since they are removable. The existing literature illustrates that the mean duration of objectively measured wear was considerably lower than stipulated wear time among all removable appliances. Moreover, patient compliance was found to be better in the early stages of CAT $[19,31]$.

With understanding of importance of patient compliance during CAT, its weakness linked with removability and advantage of fixed orthodontic devices for particularly difficult tooth movements, we can summarize:

1. patient compliance can be improved with aesthetical and comfortable device

2. combination of CAT and fixed orthodontic devices can result in optimized patient adherence to therapy reducing the time required to wear class II elastics 
3. treatment effectivity can be improved by employment of fixed appliance for difficult tooth movements

This kind of combined clinical approach has been named hybridization of aligner therapy [19]. Among others, temporary tooth-borne distalization devices are the most popular hybridization approaches in CAT. Often, they are not intended for the whole treatment period and are used effectively until desired tooth movement is achieved. The application of forces in such distalizing appliances (distalizers) could be from buccal region, palatal region, or both [19].

Several types of molar distalization appliances (distalizers) are presented in the orthodontic literature, such as the Carriere Motion 3D Appliance (CMA) (Henry Schein Orthodontics, Carlsbad, CA, USA), the Pendulum device, and the Distal Jet appliance (Figure 2).

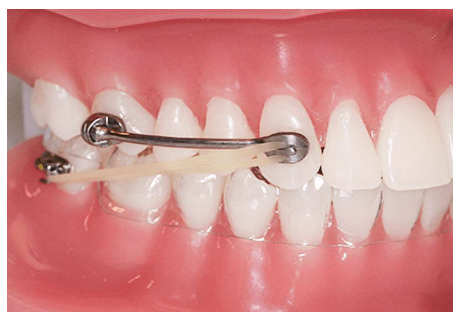

(a)

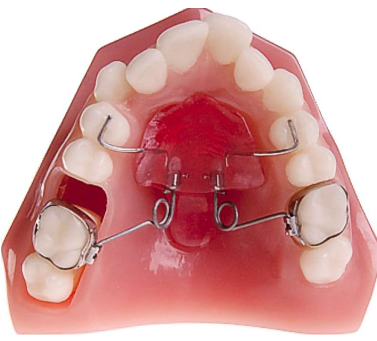

(b)

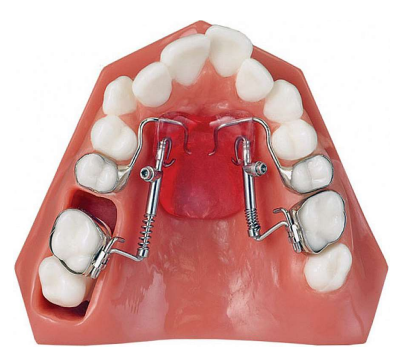

(c)

Figure 2. Examples of molar distalization appliances - distalizers (a) Carriere Motion 3D Appliance (CMA); (b) Pendulum appliance; (c) Distal Jet appliance.

Distalizers shown on the Figure 2, are believed easy to mount intraorally and can promote distal movement of the maxillary molars without the effect of maxillary orthopedic restriction $[19,32]$. Nevertheless, most of these distalizers show undesirable reciprocal anchorage loss in the premolars and incisors during distal molar movement [19, 33]. Furthermore, molar tipping is frequently observed in most of the cases. The Distal Jet appliance (Figure 2c) is composed of two bilateral tubes joined to a Nance appliance. A bayonet wire is inserted into the lingual sheath of the first molar bands [19]. On the tube there is a stainless-steel coil spring and a clamp. The clamp can slide toward the molar and be tightened to compress the coil. The force exerted by the spring begins at $150 \mathrm{~g}$ and decreases as space is opened [34]. The Pendulum appliance (Figure $\mathbf{2 b}$ ) was introduced by Hilgers in 1992 [35] and is still one of the most used distalizing devices. It is a fixed appliance composed of a plastic pad-contacted palatal rugae. The distalizing force is produced by beta-titanium springs that extend from the palatal acrylic and fit into lingual sheaths on the molar tube. Both the Distal Jet and Pendulum appliances produce an increase in the face vertical dimension due to a backward rotation of the mandible [19, 36-38]. These vertical changes comprise a slight opening of the mandibular plane angle (about 1 degree) and an increase in lower anterior facial height $(2.2-2.8 \mathrm{~mm})[19,39]$.

Ghosh and Nanda reported that the increase in lower anterior facial height was significantly greater in patients with higher pretreatment mandibular plane angles [19, 40]. The increased lower facial height and mandibular plane angle could have resulted from driving the molars back into the "wedge." These results suggest that the Pendulum may be contraindicated in patients with excessive lower facial height and/or minimal overbite $[19,38]$. Similar results were reported for the Distal Jet appliance $[19,36]$. The maxillary molar distalization obtained with those appliances is characterized by a great amount of molar distal tipping - in average more than 10 degrees [19, 33].

Whereas the Distal Jet produces a labial tipping of the upper incisors as a outcome of the uncontrolled counterforce acting on the premaxillae, the Pendulum appliance showed a more controlled inclination of the upper incisors with a mild crown buccal tipping. 
CMA (Figure 2a) consists of two rigid bars attached bilaterally to the maxillary canines and first molars. The canine pad with a built-in mesial hook used for placement of intermaxillary elastics is bonded to the anterior third of the clinical crown. Posteriorly, the molded pad with a ball-and-socket joint is bonded to the first molar at the center of its clinical crown to facilitate molar derotation and distalization [19, 41-43].

The activation of this appliance is obtained by the use of two types of elastics: the first one being $0.25 \mathrm{in}, 6 \mathrm{oz}$; the second one $0.19 \mathrm{in}, 8 \mathrm{oz}$, to be used from the second month of treatment until the molar and canine class I relationships are established. Elastics should be worn together with CAT the whole day. This means the CMA could be without elastics only 120 minutes per day maximum. Elastics shall be changed three times per day [44].

The principle of this appliance is similar to a cantilever-based fixed appliance previously shown by Nanda [45] who described the system as an effective way to correct molar class II in nongrowing patients [19].

Previous retrospective clinical studies demonstrated the possibility of obtaining a maxillary molar distalization between 1.6 and $5.1 \mathrm{~mm} \mathrm{[44]} \mathrm{with} \mathrm{the} \mathrm{mean} \mathrm{amount} \mathrm{of} \mathrm{molar}$ tipping not exceeding 3.7 degrees when CMA was used in combination with fixed appliances as anchorage units on the lower arch [44]. Furthermore, the treatment time had a mean duration of about 4 to 5 months $[19,44]$.

There is a lack of high-quality data supporting or opposing the use of CMA. In another retrospective study in which CMA effects were compared to other class II correction methods, CMA showed the same results obtained with class II elastics in terms of molar distalization but in less time [46]. There is no clinically noteworthy skeletal correction induced by CMA in growing patients. CMA can be applied to treatment of mild to moderate Class II dental malocclusion over 6 months on average, though the total treatment time may be prolonged due to several side effects [46]. One clinically important effect of treatment with CMA appeared in the lower anterior facial height that was associated with a significant increase in the mandibular plane angle $[19,47]$. The results of this study published in The Angle Orthodontist shown that the CMA is an effective way of correcting the sagittal component of Class II malocclusion within the first half-year of treatment. Proclination of the lower incisors resulting from the class II elastics mechanics was observed and resulted in a significant amount (4.2 degrees) [19, 41].

All the tooth-borne appliances mentioned above, create some side effects that need to be controlled during the hybrid aligner therapy. Excessive upper and lower incisor proclination could be difficult to control with aligners. According to Rossini et al., buccolingual tipping and torque control of upper incisors have a mean accuracy of about $50 \%$ of the planned movement $[18,19]$. The proclination of lower incisors resulting from the use of CMA could be controlled using active aligners on the lower arch and applying a lingual radicular torque on the lower incisors of at least 5 degrees. Additional side effect that can occur using tooth-borne distalization devices is the rotation of the occlusal plane due to the increase of the vertical dimension [19].

Research published by Khosravi et al. [48] regarding overbite management with Invisalign aligners indicated that overbite correction is mostly related to anterior teeth movement without any significant posterior intrusion or extrusion $[19,48]$. Also as was described by Ravera et al. [22] bite block effect of the aligners is causing an intrusive effect on posterior teeth of $0.5 \mathrm{~mm}[19,22]$. A similar value $(0.6 \mathrm{~mm})$ was described by Mantovani et al. [49] Therefore, only the 0.5- to 0.6-mm bite block effect should be considered to counteract the increase of the vertical dimension produced by tooth-borne distalization devices (average increase 2-3 $\mathrm{mm}$ ) [19].

Based on these comprehensive considerations summarized above, it can be stipulated that tooth-borne distalization devices should be avoided in orthodontic patients with marginal overbite and also in patients with excessive lower facial height. Orthodontists should be aware of the existing evidence related to the partial control of posterior intrusion, overbite correction, and buccolingual inclination provided by CAT [19]. 
The unilateral application of the Carriere Distalizer is possible and can be utilized for nonextraction Class II treatment [51].

The Carriere® Motion 3D ${ }^{\mathrm{TM}}$ appliance (CMA; Henry Schein Orthodontics, Carlsbad, Calif) has become more popular during the past decade as a versatile intermaxillary Class II corrector. The clinical principle of Class II correction using the CMA is based on establishing a Class I relationship at the beginning of treatment when patient compliance is high and before initiating the correction of the position and alignment of individual teeth with fixed appliances (or clear aligner therapy).

CMA was introduced by its developer, Luis Carriere, in 2004 as the Carriere ${ }^{\circledR}$ Distalizer $^{\mathrm{TM}}$, the renamed Carriere ${ }^{\circledR}$ Motion $3 \mathrm{D}^{\mathrm{TM}}$ appliance consists of two rigid bars bonded bilaterally to the maxillary canines and first molars. As described earlier, the canine pad with a hook attachment for placement of intermaxillary elastics is bonded to the anterior third of the clinical crown.

Intermaxillary elastics are anchored to the mandibular molars. The protocol for mandibular anchorage includes the use of a removable Essix-type clear (invisible) retainer that has been adjusted posteriorly to accommodate the bonded buccal tubes or hooks. Other methods of mandibular anchorage can embrace a lower lingual arch, temporary anchorage devices, and fixed appliances [19].

Activation of the appliance is with heavy-force $(6 \mathrm{oz}$ and $8 \mathrm{oz})$ Class II elastics with anchorage provided by the invisible lower retainer. Full-time wear of intermaxillary elastics (22 hours) is required. Phase I treatment typically takes 5-8 months to complete, given good compliance. In the adolescent patient, the CMA was shown to be more comfortable for the patient to wear, offered a more positive overall experience, and had fewer negative comfort-related side effects compared with other appliances for Class II treatment.

Regarding the long-term treatment stability: Dental and skeletal treatment results of distalization accomplished with the Carriere Distalizer appliance followed by fixed appliance therapy displayed minor changes 4 years after treatment [52].

Publication about CMA modifications from 2021 from Wilson B et al. in The Angle Orthodontist came to conclusion that shorty CMA achieved Class II correction similarly to the standard CMA, with less change in overjet and distal tipping movement of the maxillary canines. In this study both standard and shorty versions of CMAs had the posterior bracket pad bonded to the maxillary first molar with a ball and socket joint to allow for tipping and rotation of the maxillary molar. The anterior bracket pad, which has a hook, is bonded to the maxillary canine in the standard CMA and to the maxillary first premolar in the shorty CMA [53]. The simple design makes CMAs more comfortable than the Forsus appliance $[53,54]$.

With the help of CBCT 3D analysis we know, that the CMA usually corrects Class II malocclusion through distal tipping and rotational movement of maxillary canines and molars and corrected mesial tipping of mandibular molars. Significantly more mandibular molar mesial movement and maxillary incisor flaring were observed in patients with skeletal Class II [55].

This introduction placed this study in a broad context with the clinical effects of CMA and its modifications, principles and comparison to other orthodontic appliances for distalization. All of them are mostly from prefabricated materials and allow relatively limited personalization usually directly during the process when applying on the patient. With possibilities sourcing from digital workflows and 3D printing we can introduce a method to create a fully personalized 3D printed distalizer based on digital intra-oral scan.

To date, no other study has elaborated on the possibilities of biocompatible 3D printing of customized orthodontic auxiliaries like distalizers. Neither any study focused on hybrid class II treatment with CAT nor introduced such a novel method of additive manufacturing of custom distalizer for every patient. Publications regarding 3D printing of aligners or surgical splints are irrelevant to this topic. Research regarding 3D printed material application in orthodontics is mostly oriented on 3D printing of clear aligners [56 58]. 
The current state of the research field is stated above and possible reasons why such an approach was not introduced yet could be:

1. Only recently a wider range of class IIa biocompatible dental resins for 3D printing has been introduced and fully certified in Europe

2. Interdisciplinary approach might be necessary to invent and establish this practice that includes 3D digital scanning, 3D software modelling, 3D biocompatible printing and orthodontic knowledge of biomechanics

To highlight why is this study important is the fact that until now, no other study has published such novel and affordable method of employment of 3D printing from new dental resins to materialize effective orthodontic auxiliary such as distalizer. It will inspire orthodontists to take advantage of intraoral scans they do now routinely and employ them in this practice of creation of applicable auxiliaries not necessarily only comfortable distalizers. The purpose of this the work is to show the whole procedure of simple 3D modeling, printing and application of distalizer (CDA-like) for hybrid class II CAT.

The secondary objective was to compare two popular biocompatible materials and evaluate their clinical performance as well as the patient appraisal.

The significance of this work lies in the empowerment of orthodontists to develop their own customized auxiliaries for hybrid treatments or even in wider clinical context. Returning treatment individualization back to their hands with the opportunity to fully personalize the treatment of their patients as they did in the past in the times of remarkable "wire-benders" and every appliance was individualized. The goal of this described procedure is the materialization of personalized shape of biomechanical auxiliary to increase the speed or comfort of the orthodontic treatment.

What can be stressed from this new approach is, that resulting 3D printed distalizer is unique in every patient, with appropriate length, curvature and most notably its terminal pads have individualized base for bonding. This means that distalizer's surfaces intended for bonding are complementary to particular surfaces of teeth, usually vestibular and more cervical parts of upper canine and molar. Biomechanics of personalized distalizers presented in this article is closest to the Carriere ${ }^{\circledR}$ Motion $3 \mathrm{D}^{\mathrm{TM}}$ appliance as it is representative of the most effective tooth-borne distalizers with dental effect.

To keep the introduction comprehensible to scientists outside orthodontic field of research the principal conclusions could be that: individual distalizer on the principle of Carriere Distalizer can be modeled and printed with small effort to achieve excellent clinical objectives and high patient comfort. This proves that it is possible to implement the biocompatible Additive Manufacturing (AM) and 3D modeling in better personalization of other orthodontic auxiliaries like power-arms, power-caps, retainers and many other applications (Figure 3).

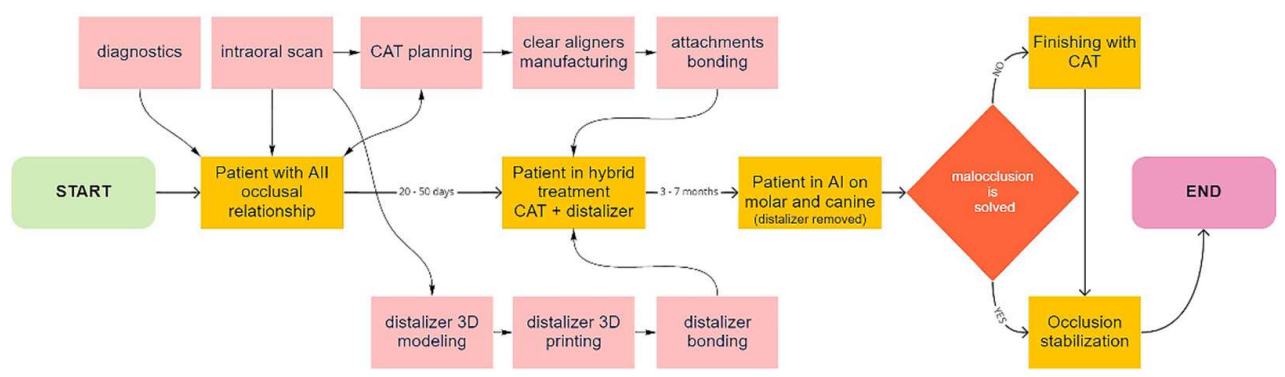

Figure 3. Scheme describing new digital workflow for orthodontist utilizing certified biocompatible dental resins for 3D printed personalized distalizers for hybrid treatment.

This article evaluates two photopolymers within application of a novel method of design and manufacturing of a personalized distalizer. Materials and distalizers were clinically evaluated on the complete distalization treatment procedures on 12 patients with unilateral class II occlusal relationship. 12 patients were chosen upon strict criteria from 
the total set of 387 monitored patients. Material distribution was random. Objective for the strict criteria was elimination of possible bias caused by clinical differences.

To properly design, print and apply personalized distalizer for hybrid treatment, the more complex clinical picture and target shall be comprehended for each particular case (Figure 4). Example shows that not necessarily whole extent of distalization trajectory must be achieved only with distalizer. Its combination with CAT allows wider scope of approaches for the orthodontist (Figure $1 \mathrm{a}, \mathrm{b}, \mathrm{c}$ ).

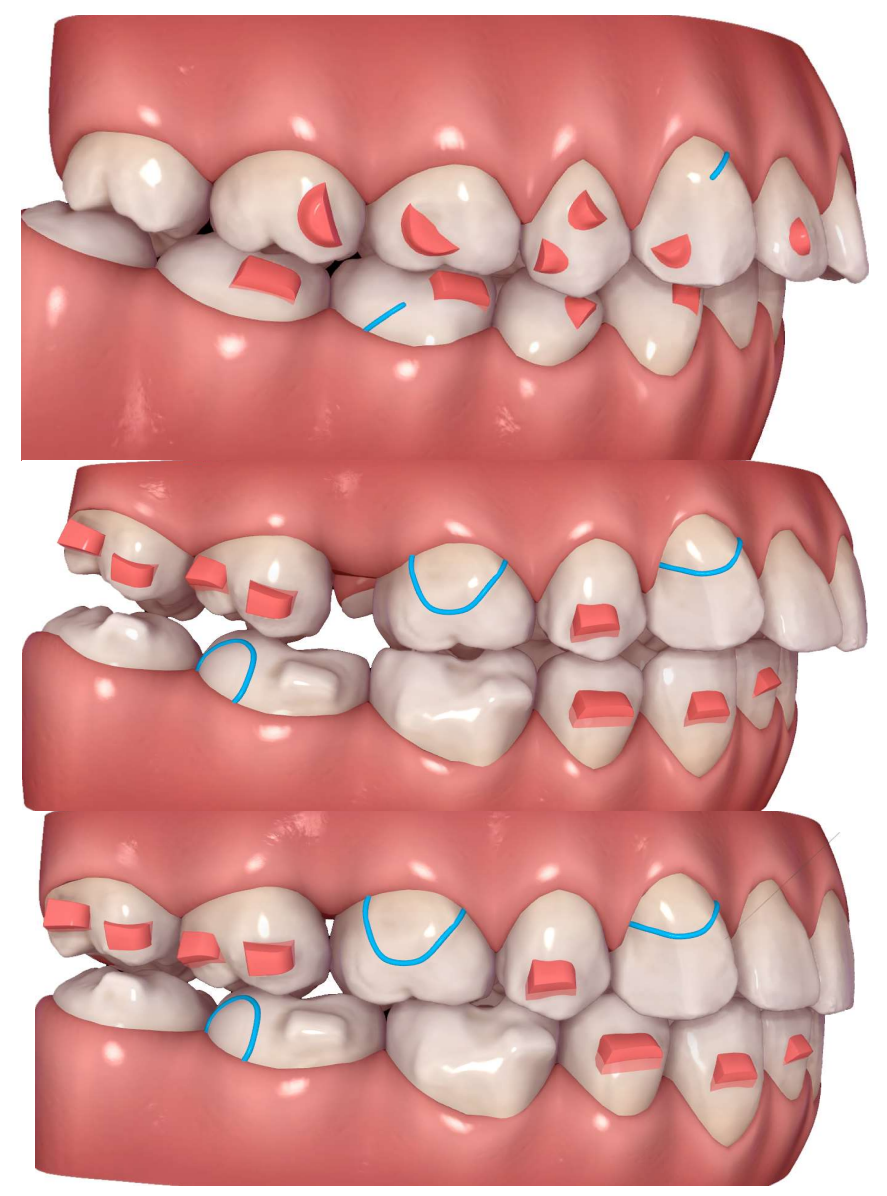

(a)

(b)

(c)

Figure 4. Planning of personalized unilateral distalizer for upper teeth distalization in clinical hybrid approach starts with complex understanding of the clinical situation, treatment target and decision which tasks will be managed by CAT and which by distalizer (a) Initial setup with class II. elastics in the CAT (Invisalign ${ }^{\circledR}$ ) to achieve distalization of two terminal molars 18 and 17; (b) Situation evaluated for 3D printed personalized distalizer application with similar biomechanical effect to Carriere ${ }^{\circledR}$ Motion $3 \mathrm{D}^{\mathrm{TM}}$ distalizer Appliance (CMA). The "Button cutouts" in the aligner on teeth 13 and 16 are intended for 3D printed distalizer custom base fixed adhesion on tooth surface; (c) Planned clinical target of hybrid treatment with CAT + distalizer with $15 \%$ overcorrection; 


\subsection{Patients}

To fairly compare both materials clinically, the strict criteria were defined to minimalize possible bias. Bias could result from non-homogenous group affected by growth or skeletal discrepancies or extreme extends for distalization or application of different types of CAT or poor monitoring caused by patients 'non-compliance. Patients for evaluation of the described tooth-borne hybrid treatment approach with class II unilateral malocclusions were chosen by following selection criteria:

1. Patient must be adult

2. Unilateral class II occlusion measurable on molars and canines with class I relationship on the opposite side and no skeletal class II values (Figure 4)

3. Skeletal cephalometric values of $\mathrm{SNA}=81^{\circ} \pm 3^{\circ}, \mathrm{SNB}=78^{\circ} \pm 3^{\circ}, \mathrm{ANB}=3^{\circ} \pm 2^{\circ}$

4. Target of planned linear distance of frontal cusp of upper molar translation (distalization and distorotation) had to be between 2,5 and $6 \mathrm{~mm}$

5. Patient was compliant with Dental monitoring on a weekly basis

6. Patient had distalizer combined with CAT (all patients were using Invisalign type of appliance)

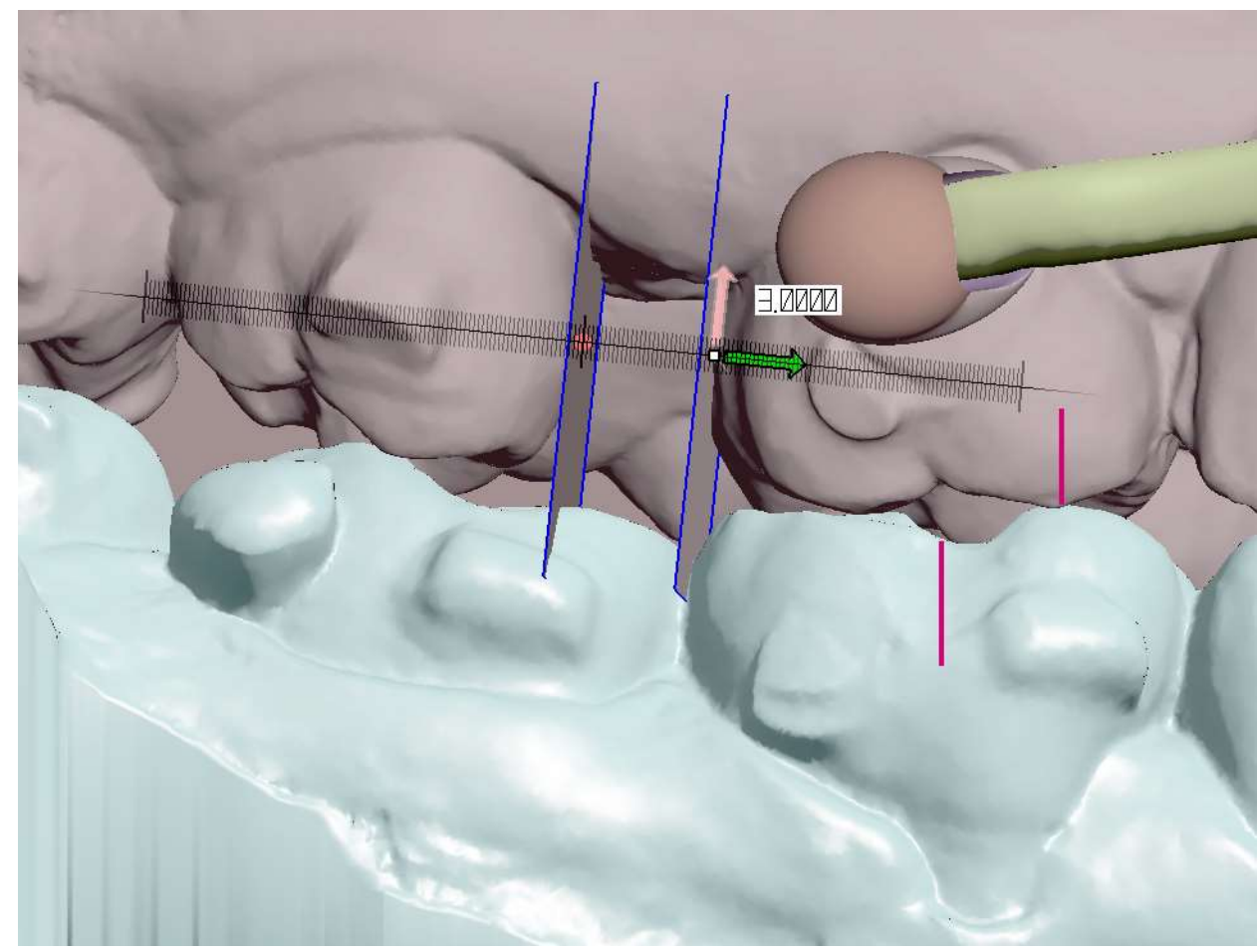

Figure 5. Digital measurement of planned clinical target of distalization with disto-rotation of the upper first molar was realized on the digital intra-oral scan.

Reasons for these selection criteria is described in more detail in the Introduction chapter. Especially the exclusion criterium for skeletal class II was based on published variability of distalizers effects in between skeletal class I and class II cases. As well exclusion criterium for planned extend of upper molar distalization over $6 \mathrm{~mm}$ and under $4 \mathrm{~mm}$ allowed better harmonization of patient sample. As the goal of this research was to compare distalizer clinical performance between two similar materials, the potential bias caused by differences in distalization difficulty or the setup might simulate differences between both groups.

After application of these criteria from 385 A.I. monitored patients only the final 12 adults in first skeletal class that were acknowledged with Invisalign treatment were selected as suitable. These patients were randomly divided into two groups according to type of dental resin used for distalizer manufacturing. All patients were monitored on a 
weekly basis by video-scans evaluated by artificial intelligence algorithm and no significant differences in compliance were observed in these patients. Dental monitoring was able to identify immediately a drop or damage of orthodontic auxiliary (distalizer) so we were not depended on patient self-reporting.

\subsection{Materials, handling and 3D printing and post-printing protocols}

Two long-term biocompatible composite resins, intended for 3D printing, were chosen to be compared:

1. Dental LT Clear Resin (RS-F2-DLCL-02 from Formlabs) [59, 61, 62]

2. Denture Teeth Resin (FLDTA101,PKG-RS-F2-DT Formlabs/Dentca) $[60,61,63,64]$

The reason for choosing these two materials was to evaluate if such a small differences of material properties does have significant impact on the clinical performance of 3D printed distalizer. Second reason was to evaluate the patient feedback whether the transparent or the opaque distalizer is considered more aesthetical.

Dental LT Clear Resin (RS-F2-DLCL-02 from Formlabs) [59] is designed for hard splints and retainers with high resistance to fracture and wear. This resin is Class IIa biocompatible. 3D printing layer accuracy of this material is only 100 microns which is sufficient for expected details of distalizer, however was relevant to compare to second material which was printed on resolution of 50 microns. This biocompatible photopolymer resin is intended for Form 2 3D printer. Advantage of this material is that it is highly durable and resistant to fracture and has high optical transparency, with resistance to discoloration over time.

Post-processing after 3D printing:

After printing the distalizer, it must be washed and the support removed. Dental LT Clear Resin V2 shall be washed in IPA with a concentration of 99\% or higher to comply with biocompatibility regulations. In our protocol we have washed the clear distalizer parts for 15 minutes, then removed and soaked in fresh IPA for 5 minutes. For optimum mechanical properties, it mustn't be washed for more than 20 minutes. After washing is must be light cured ideally in the Form Cure that helps 3D printed parts achieve their highest possible strength and stability. Optimal post-curing settings depend on equipment and the geometry of the part. In our case the recommended settings can be applied [61]. This means that distalizer from material Dental LT Clear V2 shall be light cured for 60 minutes in temperature of $60{ }^{\circ} \mathrm{C}$. This cure setting ensures that it achieves both biocompatibility and optimum mechanical properties. This material has been updated in 2020 and clinicians shall remember this. The FLDLCL02 resin (available since 6/2020) is a new version of FLDLCL01(was available since 10/2017). This new resin was used in this research and in comparison, to older version of this composite material it has reduced yellowing, improved durability a higher resistance to fracture. Further technical details about this material are summed up in the Table 2 . As well as its technical and safety sheets online $[59,62]$ 
Table 2. Material properties of LF Dental clear V2

\begin{tabular}{ccl}
\hline Mechanical Properties & $\begin{array}{c}\text { Post-Cured } \\
\text { Post-cured Dental } \\
\text { LT Clear resin (V2) }\end{array}$ & \multicolumn{1}{c}{ Method } \\
\hline Ultimate Tensile strength & $52 \mathrm{MPa}$ & ASTM D638-10 (type IV) \\
Young`s modulus & $2080 \mathrm{MPa}$ & ASTM D638-10 (type IV) \\
Elongation & $12 \%$ & ASTM D638-10 (Type IV) \\
Flexural Strength at 5\% Strain & $84 \mathrm{MPa}$ & ASTM D790-15 (Method B) \\
Flexural Modulus & $2300 \mathrm{MPa}$ & ASTM D790-15 (Method B) \\
Hardness Shore D & $78 \mathrm{D}$ & ASTM D2240-15 (Type D \\
Water Absorption & $0.54 \%$ & ASTM D570-98 (2018) \\
\hline
\end{tabular}

Denture teeth Resin (FLDTA101, PKG-RS-F2-DT from Formlabs/Dentca) [60, 61, 63]

Formlabs Denture Resins are certified biocompatible materials for 3D printing digital dentures and their parts. This resin is also Class IIa biocompatible. 3D printing layer accuracy of this material is 50 microns which is better than in previous transparent resin and better for expected details of distalizer. It is also available in all tooth-color shades. Upon research and inquiring the official Formlabs support we have found out, that this resin is exceptionally not their proprietary and its original manufacturer is company Dentca, Inc. located in Torrance, CA, United State. Composition of this resin matches DENTCA Denture Teeth resin an FDA cleared biocompatible photocurable material with performance and strength matching conventional acrylic. DENTCA is a manufacturer of medical and healthcare devices focused on denture base and teeth products and was first to receive FDA approval for such 3D printing resins.

Form wash setting for this application were different than in previous material. In previous s transparent resin, the wash time was 20 minutes, in this denture-teeth resin it is only 10 minutes.

Form Cure settings for this material is specific. In our protocol we have followed the official guidelines $[63,64]$ and we have used a glass container with glycerin. After preheating the glycerin to $80^{\circ} \mathrm{C}$ in Form Cure, we have used heat resistant own apparatus to keep the distalizers fully submerged in the glycerin, and kept the container inside Form Cure for 30 minutes. After the first 30-minute post-cure, we have flipped the small distalizers to the opposite side. Post-cure again for 30 minutes.

Composites are complex materials, and finding the right one for our specific application requires balancing multiple attributes. Distalizer is a small part with need of rapid prototyping.

Mechanical properties od Denture teeth resin is Flexural Strength $>65 \mathrm{MPa}$ (method ISO 20795-1) and density of the material of $1.15 \mathrm{~g} / \mathrm{cm} 3$ (method ASTM D792-00). Denture Base ISO Standard used is EN-ISO 20795-1:2013 (Dentistry - Base Polymers - Part 1: Denture Base Polymers) summarized in the (Table 3).

Table 3. Known properties of Denture teeth Resin (Formlabs, Dentca)

\begin{tabular}{|c|c|}
\hline Properties & $\begin{array}{c}\text { Post-Cured } \\
\text { Denture teeth Resin (shade A2) }\end{array}$ \\
\hline Density of the material ${ }^{1}$ & $1.15 \mathrm{~g} / \mathrm{cm}^{3}$ \\
\hline Flexural Strength 2 & $90 \mathrm{MPa}$ \\
\hline Relative worn volume $\%$ of $\Delta \mathrm{V}^{3}$ & $70 \%$ \\
\hline
\end{tabular}

Material properties can vary with part geometry, print orientation, print settings, and temperature. Data in Tables 2, 3 and 4 refer to post-cured properties obtained after exposing parts to 108 watts each of Blue UV-A $(315-400 \mathrm{~nm})$, in a heated environment at $80{ }^{\circ} \mathrm{C}\left(140^{\circ} \mathrm{F}\right)$ and $1 \mathrm{hr}$, with six 18W/78 lamps (Dulux blue UV-A). [59 - 65] 
Table 4. Chemical composition of Dental LT clear and Denture teeth Resin (Formlabs, Dentca)

\begin{tabular}{|c|c|c|c|}
\hline Post-cured Dental LT Clear resin (V2) & $(\% \mathrm{w} / \mathrm{w})$ & $\begin{array}{l}\text { Post-cured Den- } \\
\text { ture Teeth Resin } \\
\text { (shade A2) }\end{array}$ & $(\% \mathrm{w} / \mathrm{w})$ \\
\hline $\begin{array}{l}\text { 7,7,9-trimethyl-4,13-dioxo-3,14-dioxa-5,12- di- } \\
\text { azahexadecane-1,16-diyl bismethacrylate }\end{array}$ & $50-75$ & $\begin{array}{l}\text { Proprietary methacrylate } \\
\text { monomer }\end{array}$ & $40-60$ \\
\hline 2-hydroxyethyl methacrylate & $10-20$ & Diurethane dimethacrylate & $30-50$ \\
\hline $\begin{array}{l}\text { Reaction mass of Bis(1,2,2,6,6-pentamethyl-4-pi- } \\
\text { peridyl) sebacate and Methyl 1,2,2,6,6-pentamethyl- } \\
\text { 4-piperidyl sebacate }\end{array}$ & $<10$ & $\begin{array}{l}\text { Trimethylolpropane tri- } \\
\text { methacrylate }\end{array}$ & $3-10$ \\
\hline Diphenyl (2,4,6- trimethylbenzoyl) phosphine oxide & $1-5$ & Initiator & $<3$ \\
\hline Acrylic acid, monoester with propane-1,2-diol & $0.1-1$ & Stabilizer & $<1$ \\
\hline ethylene dimethacrylate & $<10$ & Pigment & $<0.7$ \\
\hline
\end{tabular}

\section{$2.33 \mathrm{D}$ printing concept and costs}

We have used Formlabs stereolithography 3D printer called Form 2 with standard setting. Its technical parameters are available online [65].

In every case, we have been printing on the highest resolution available $(0.1 \mathrm{~mm}$ for clear or $0.05 \mathrm{~mm}$ for white resin). In every case we have printed 3 clones in total, for practical reasons anticipating situation of possible damage to the distalizer, to be able to immediately replacing the damaged / debonded part. For simplicity the shade of Denture teeth resin was chosen as A2.

The approximate costs regarding prefabricated "CARRIERE Motion 3D Colors Class II Appliances Distalizer 13-30 L\&R with Ruler" on ebay is $\$ 50-\$ 60$. Printing costs per one triple-set of distalizers is approximately $\$ 15$ ( $\$ 3-\$ 5$ a part). This calculation is approximation and relates to denture teeth resin costs which are $\$ 399$ per liter. Dental LT clear costs also $\$ 399$ per liter. Costs for 3D printing with both materials does not differ significantly neither do material mechanical properties.

Toothbrush wearing test was performed by Dentca company to review the abrasion resistance of preformed DENTCA Denture Teeth compared with commercial artificial polymer teeth. Relative worn volume $\%$ of $\Delta \mathrm{V}$ to reference artificial acrylic tooth (100\%) was 70\%, This means the DENTCA Denture Teeth are less wear than the commercial artificial acrylic tooth. Stain Resistance was tested by Dentca company to review the staining resistance of preformed Denture Teeth resin compared with commercial artificial polymer teeth by soaking into two different solutions. Staining resistance of DENTCA Denture Teeth is equivalent to artificial polymer tooth.

Despite the resin manufacturing company Dentca does not publish detailed material properties of their 3D printing denture teeth resin, it has been fortunately well researched [66]. Yoo-Jin Chung et al. in their article "3D Printing of Resin Material for Denture Artificial Teeth: Chipping and Indirect Tensile Fracture Resistance" evaluated chipping and indirect tensile fracture resistance of this resin.

Both materials have similar properties however they differ significantly in their clinical appearance (one is opaque white another is transparent). Participating patients were questioned about their satisfaction with distalizer appearance / aesthetics. Patients at the end of the treatment had option to reference if the next distalizer would prefer in the same color or they would prefer another (still choosing only between white and transparent).

\subsection{Method of digital design of personalized orthodontic distalizer}

One of the goals of this paper is to demonstrate that the making of personalized distalizer is not complicated and time consuming. The whole hybrid treatment workflow is shown in the scheme on the Figure 3. For designing personalized orthodontic distalizer we have followed these steps: 
- The first step is to register intraoral situation with digital intraoral scan and exporting it in a common 3D format. For example, STL file format is suitable and can be processed in various simple 3D modeling programs like Meshmixer (from Autodesk).

- The second step is to coordinate distalizer and CAT planned effects and plan the placement of distalizer in harmony with CAT. In this research all CAT were performed using Invisalign appliances (Figure 6).

- Third step was to design the body of the distalizer, including movable joint and individual bases on the terminal pads of the distalizer.

- Fourth step was clinical application and distalizer activation followed by weeks of movement Dental monitoring.

- Fifth step was performed after achieving planned occlusal correction. Removal of the distalizer was followed by stabilization of the result.

Planning of the complex hybrid treatment requires to coordinate distalizer and CAT. Their effects shall be in synergy and the placement of distalizer might be on teeth covered with aligner or, less frequently, not. One of possible steps is overlay of intraoral scan with CAT treatment model to identify the planned aligner cut-outs for 3D printed distalizer adhesive individualized bases (Figure 6). In this paper we explain the advantage of customized distalizer on particular clinical situation (unilateral class II) and particular clinical approach (tooth-borne hybrid treatment with orthodontic distalizer and CAT).

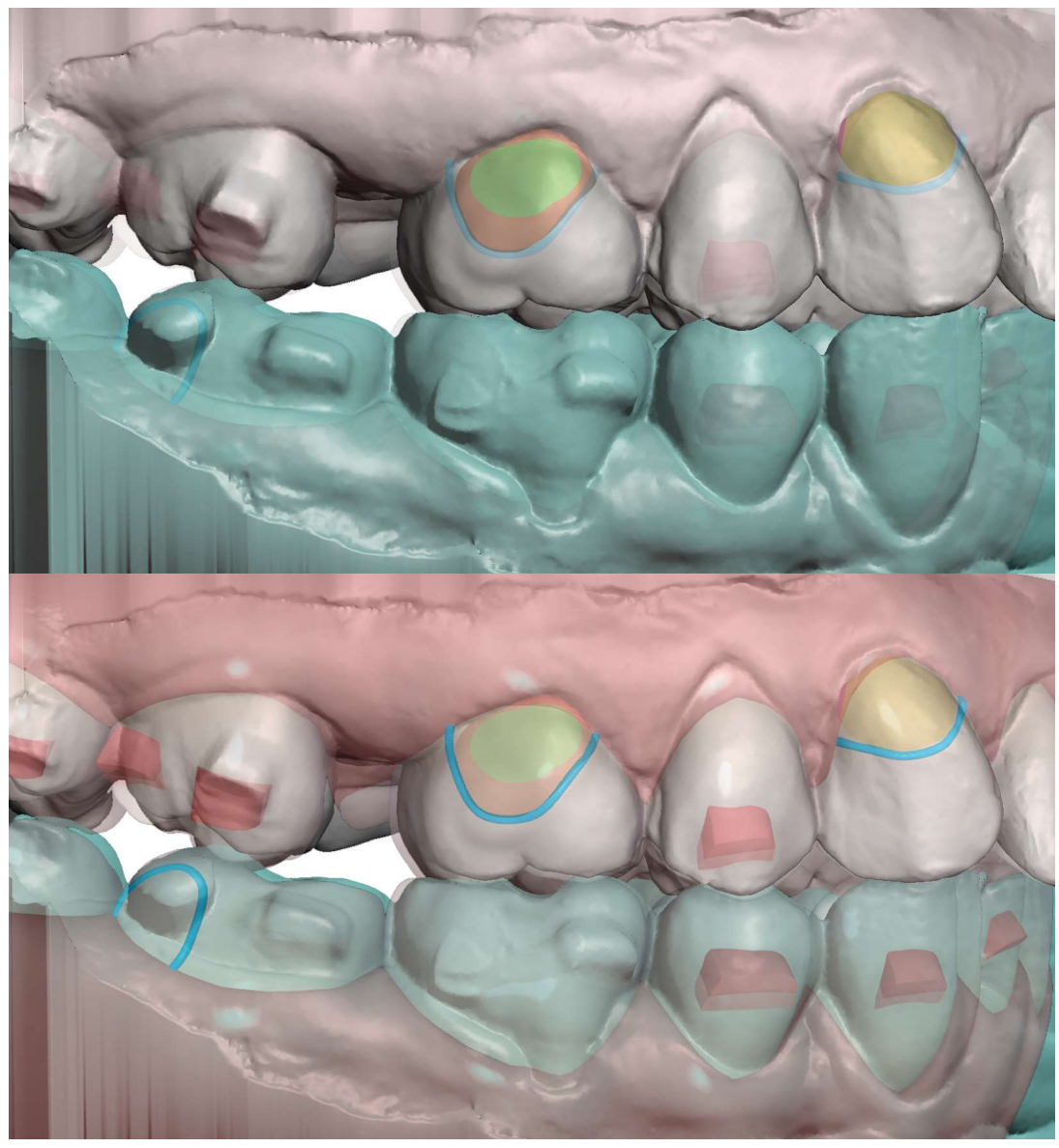

(a)

Figure 6. Overlay of intraoral scan (a) with software model from CAT planning (b) is important to identify the areas of aligner cut-outs. These will be the contacts of distalizer with teeth surfaces.

After identification of the areas for distalizer bonding; the body, joint and bases shall be designed. To start designing the distalizer the plane shall be defined on which the 
distalizer long axis will lie crossing approximately the middle of the areas intended for bonding of the distalizer. Placing 2D plane in the program like Meshmixer is sufficient. Placing and reshaping two spheres in these spots will represent future bodies of the individualized pads with bases copying the surface of the teeth in these locations (Figure 7a). After placements and resizing the spheres a contour of the distalizer body shall be drawn on the plane, keeping distance from premolar teeth surfaces of around 0.4-0.8mm (Figure $1 b)$.

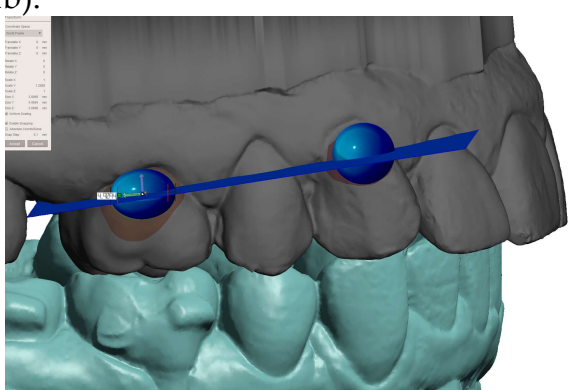

(a)

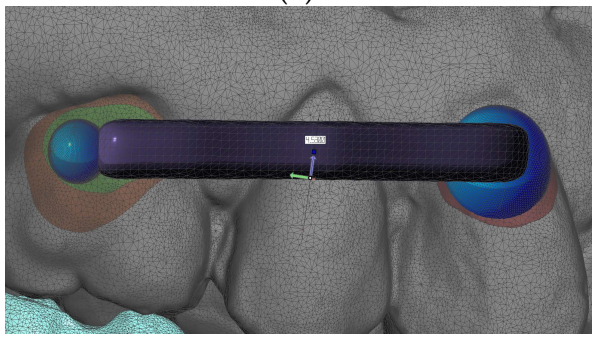

(c)

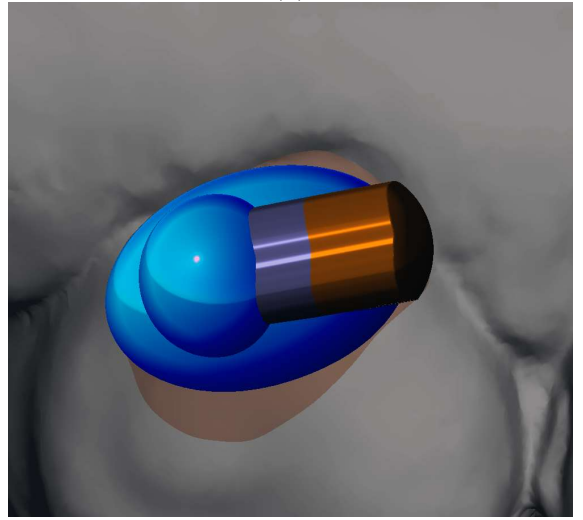

(e)

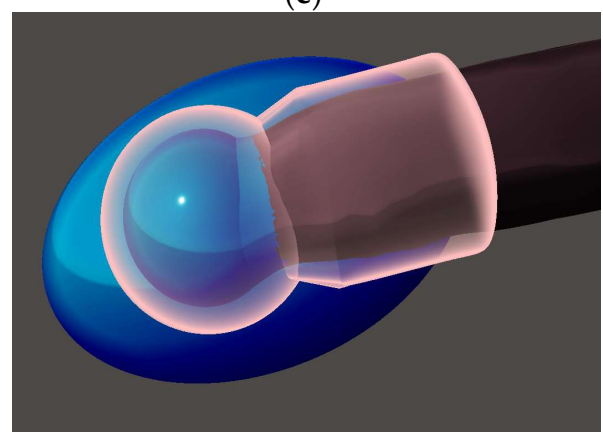

(g)

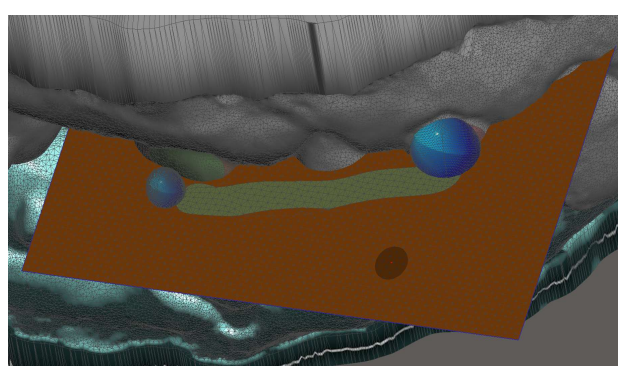

(b)

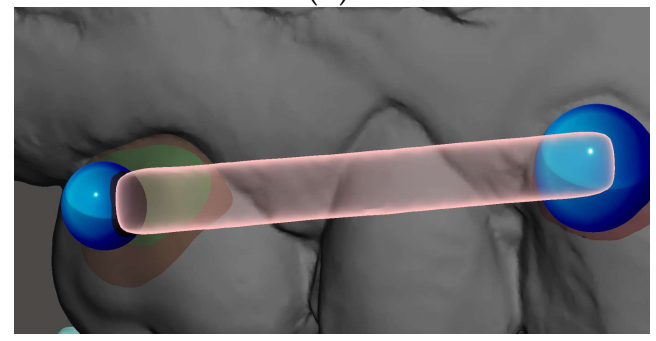

(d)

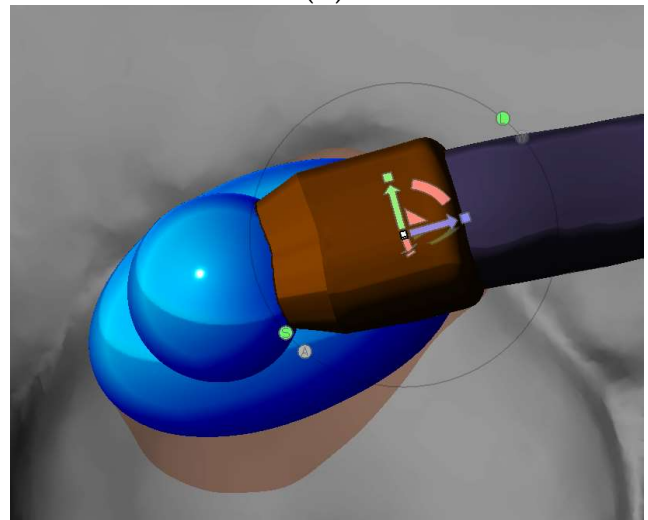

$(\mathbf{f})$

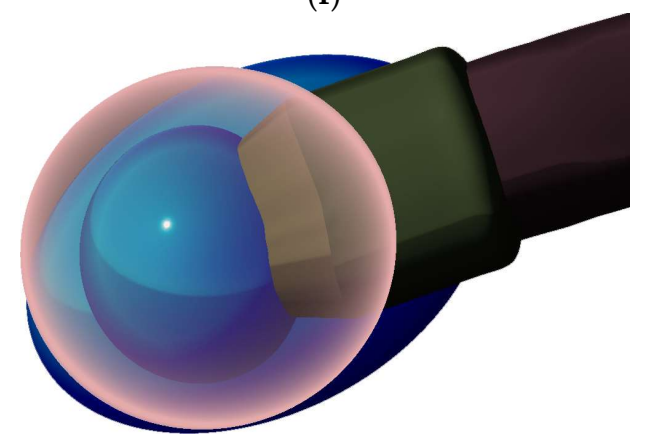

(h)

Figure 7. Method of designing personalized distalizer. (a) placing a plane for the upper border of arm of the distalizer and spheres overlaying teeth surfaces in the areas of future individualized adhesion pads; (b) Optimized border and deleting the rest of the plane; (c) 4,5 mm extrusion of the 2D plane selection creates the arm of distalizer; (d) New sphere $(\mathrm{d}=5 \mathrm{~mm})$ on the distal end of the arm represents the terminal joint of the distalizer`s arm. New sphere, with the same center is 
created with $0.1 \mathrm{~mm}$ bigger diameter and represents the future socket for the joint; (e) Extrusion of the spherical socket for the joint in the direction of the arm, liberating it movement during distorotation of the molar; (f) extending the hiatus of the distalizer's arm access to the joint socket; (g) visualization of the future joint socket and its access together with distalizer's arm distal ending with spherical joint; $(\mathrm{h})$ creation of final coverage of the joint with a new sphere resized as necessary to cover the joint socket at least by $0,6 \mathrm{~mm}$;

After definition of the arm 2D contour (Figure $7 \mathrm{~b}$ ) extrusion follows. Creating a bar that after smoothing and rounding the edges (in Meshmixer program by pressing Ctrl + F) an arm of the distalizer is created (Figure 7c). It can be directly merged with the future adhesive base on the canine. Another step is creating a new sphere with diameter of $5 \mathrm{~mm}$ with center identical to center of the terminal sphere for joint of the distalizer's arm. This sphere will represent the terminal joint socket for the distalizer's arm. Difference between both spheres (joint head and joint socket) shall be between $0.1 \mathrm{~mm}$ up to $0.2 \mathrm{~mm}$. New sphere, created will be extruded in the area of arm accessing the joint (Figure 7e, f). Spherical socket is extended in this direction to allow the distalizer 's joint movements, liberating the arm during distorotation of the molar (Figure $7 \mathrm{~g}$ ). The final step in designing the juncture of both parts of the distalizer is the creation of final coverage of the joint with a new sphere resized as necessary to cover the joint socket at least by $0,6 \mathrm{~mm}$ (Figure $7 \mathrm{~h}$ ).

Scheme on the Figure 8 describes main parts of the juncture of both distalizers parts located on the smaller part of the distalizer intended for molar placement. (1) is the final encapsulation of the joint when merged with the base (3) this creates the body of the smaller part of the distalizer, intended for bonding on the vestibular surface of the molar crown. Part (2) represents the socket of the joint, where the pressure of the distalization is applied allowing the distorotational movements of the molar. The last part (5) represents curved terminal part of the distalizer's arm entering the socket with sufficient gap around it.

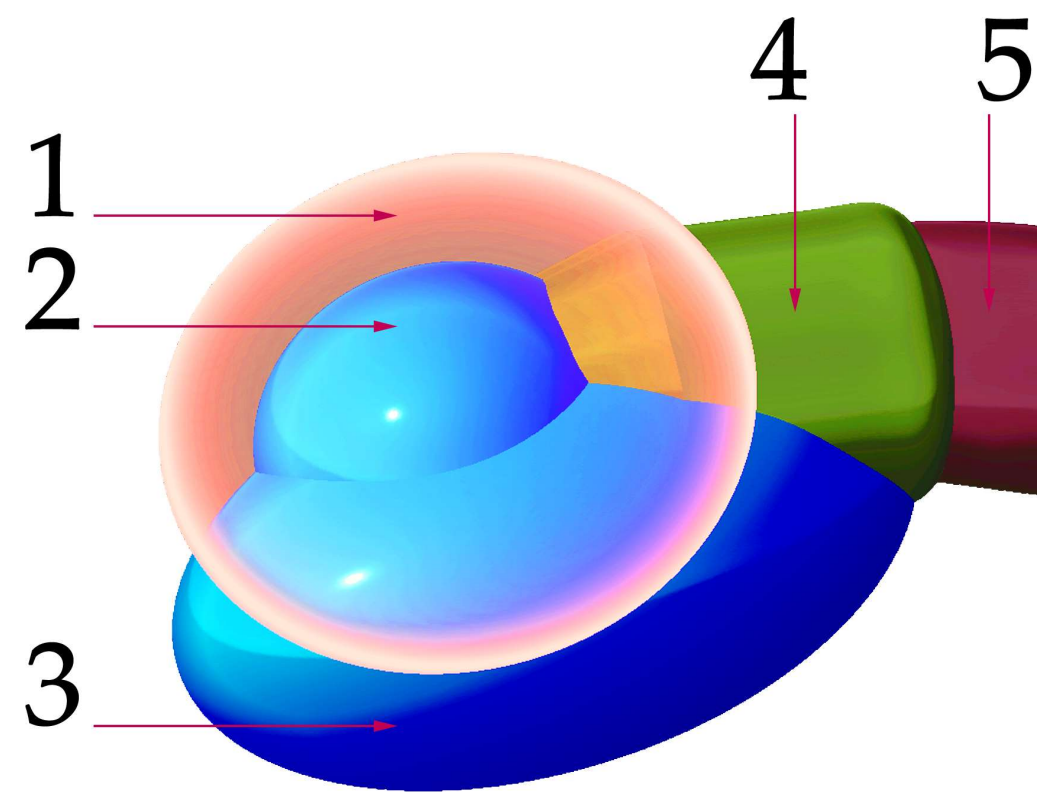

Figure 8 Main parts of the junction of both distalizers parts located on the smaller part intended for molar adhesion. (1) final encapsulation of the joint when merged with the base; (3) the body of the smaller part of the distalizer with surface complementary to the surface of the molar thus creating an individual base; (2) the socket of the joint; (4) extended neck of the accessory channel for the arm ending in the socket, liberating arm orientation during distalization and distorotation of the molar; (5) terminal part of the distalizer`s arm entering the socket with sufficient gap around it;

Figure 9 visualizes final merge of the distalizer parts creating individualized bases, arm and the socket in regards to the teeth (Figure 9a, b, c). 


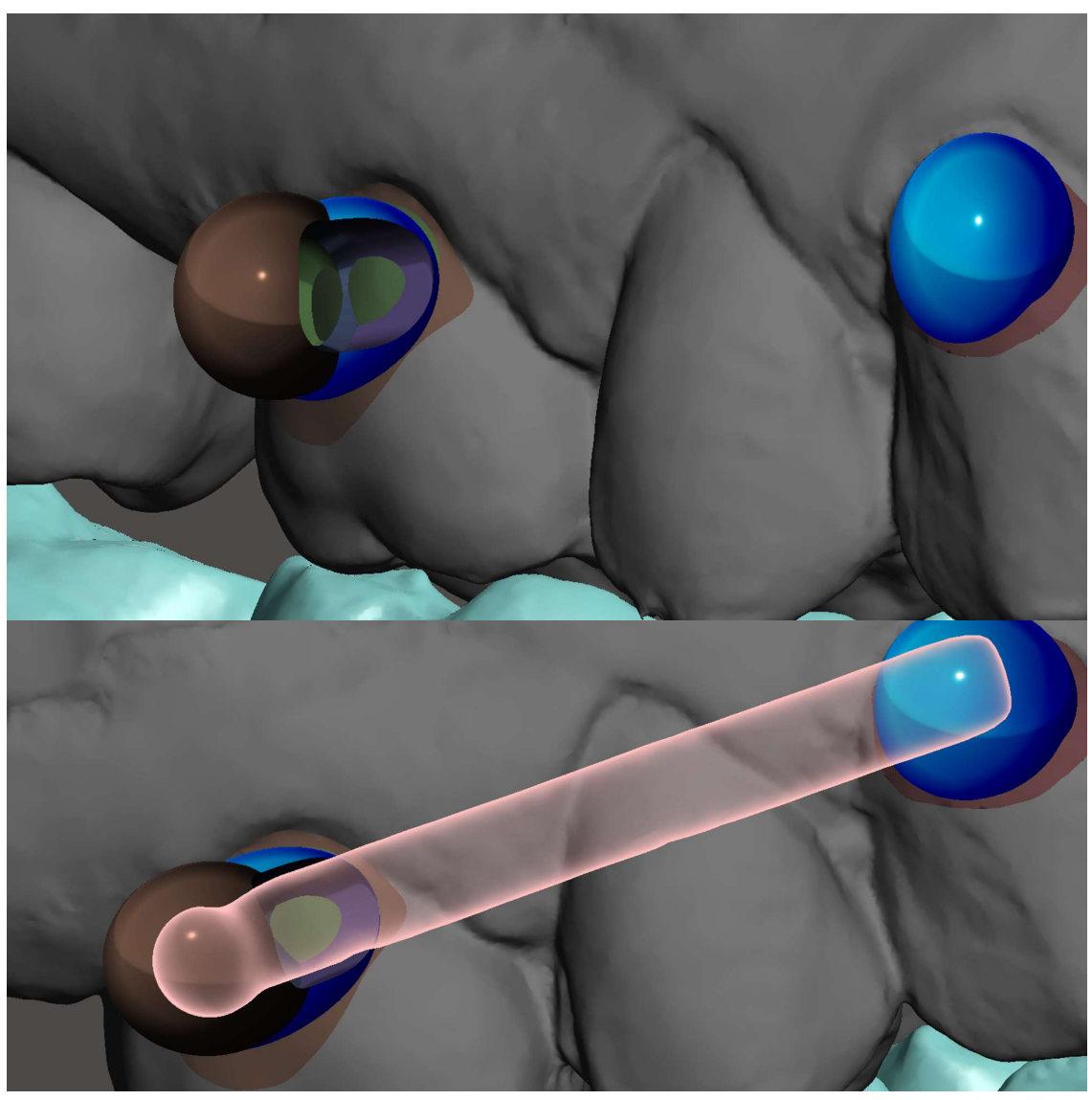

(a)

(b)

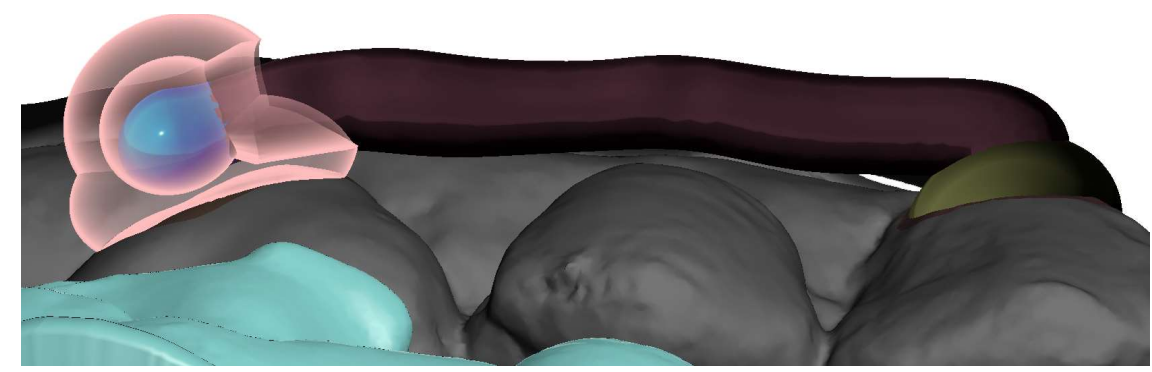

(c)

Figure 9. Visualization of the juncture of both parts of the distalizer (bigger part with the arm and joint and the smaller part with the socket). (a) Visual of the molar part of the distalizer including the socket; (b) Visualization of ending of the arm of the distalizer in the body of the distal part; (c) Profile of the layers of the distalizer`s body, socket and the joint;

Individual base of the distalizer is responsible for perfect match to tooth surface. Both ends of the connected distalizer are intended to be bonded as a fixed orthodontic auxiliary for necessary period of time during orthodontic treatment. Figure 10a shows how such individual base looks like. Figure 10b shows hiatus in the tunnel leading to the socket. Small circular step results in "click-in" function, where slight pressure is necessary to "click-in" the arm of the distalizer with spherical joint into the socket. 


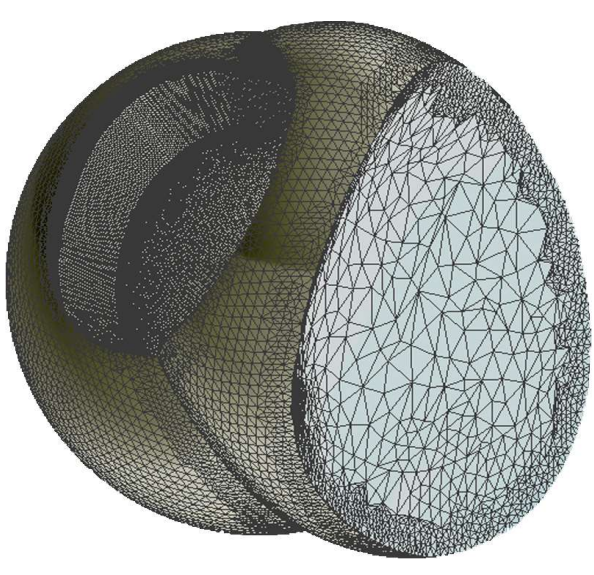

(a)

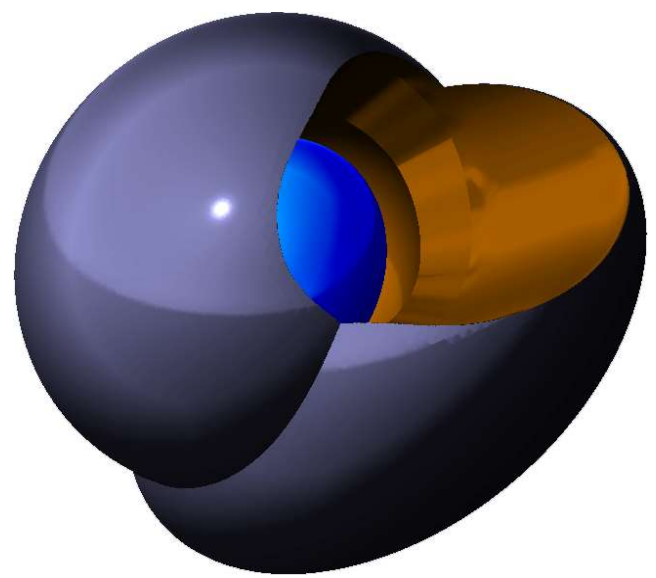

(b)

Figure 10. Vestibular and lingual side of the molar part of distalizer. (a) Wireframe appearance of dividual base that matches tooth surface in exact area that is helping in exact positioning (b) The tunnel that leads to the socket with visible hiatus - a small circular step that is causing a "click-in" function, where slight pressure is necessary to "click-in" the arm of the distalizer with spherical joint into the socket - both parts of distalizer shall be connected before bonding.

Further steps explained in the Figure 11(a - h) describe a creation of feature supporting anchorage of the elastic bands. Figure 11a shows more rounded surface in contrast with Figure 9c. Such slight adaptations can be made wherever operator considers it appropriate with goal to achieve more comfortable shape for the patient and better resiliency during chewing. Authors of this paper used program Meshmixer from Autodesk and mode Sculpt $->$ Surface $->$ brush (robust-smooth). Step in connecting area between distalizer's arm and the canine base is smoothed.

\subsubsection{Elastic bands attachment and distalizer activation}

Placement and removal of the elastic bands is performed by the patient. The elastics shall be changed for new at least 3 times per day. Activation of the distalizer is done immediately after bonding. The activation is obtained by the use of two types of elastics: the first month $0.25 \mathrm{in}, 6 \mathrm{oz}$; and $0.19 \mathrm{in}, 8 \mathrm{oz}$, to be used from the second month of treatment.

Loading with this heavy-force elastic bands $(6 \mathrm{oz}$ and $8 \mathrm{oz})$ is tooth borne from the pad on the maxillary canine to mandibular molar button or precision cut in the lower aligner.

It is expected a full-time wear from the patient ( 22 hours). There are various ways of creating a button or another form of retentive feature on the canine pad. Figure 11 shows only one of them. This method and design of attachment for elastic band, presented on Figure $11 \mathrm{~b}$ - f, we consider very practical, comfortable and resilient. Figure 11g shows arm of the distalizer with individual base intended for canine mesial - cervical part of maxillary canine. 


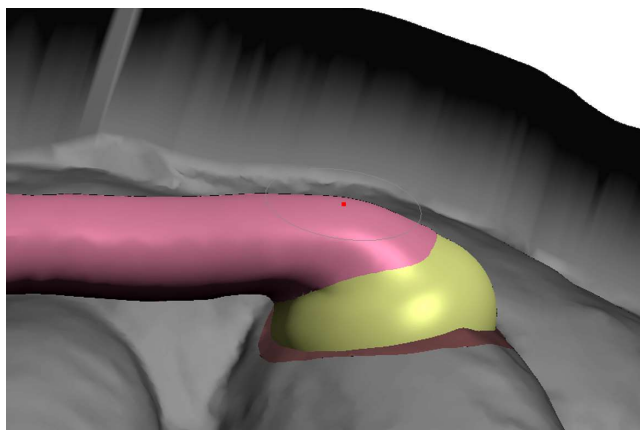

(a)

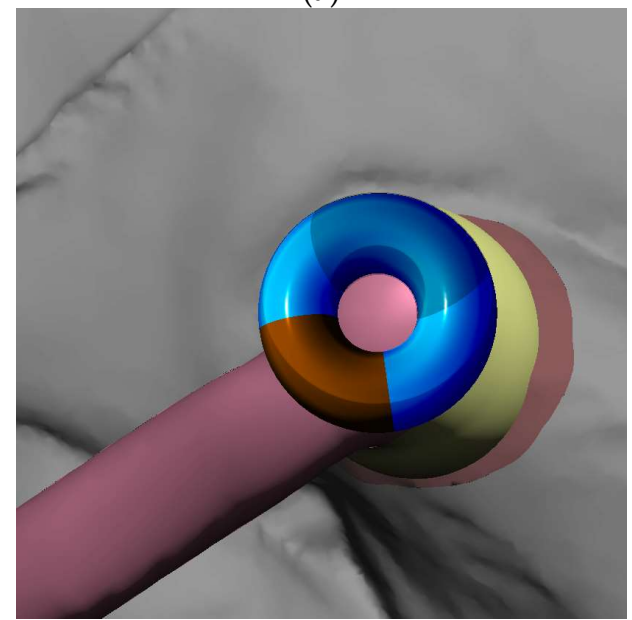

(c)

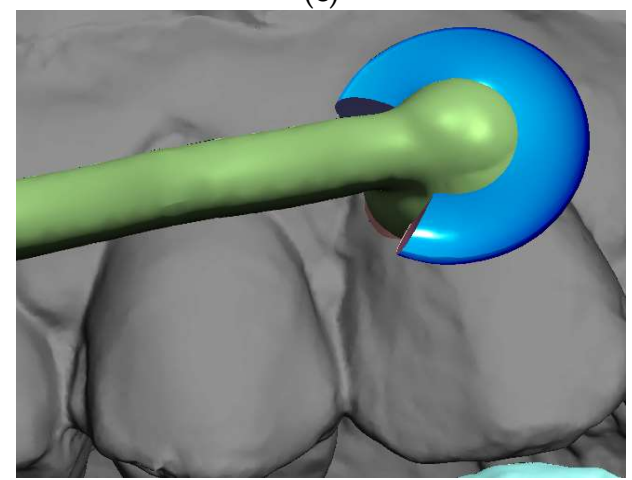

(e)

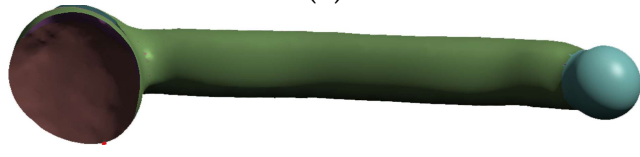

(g)

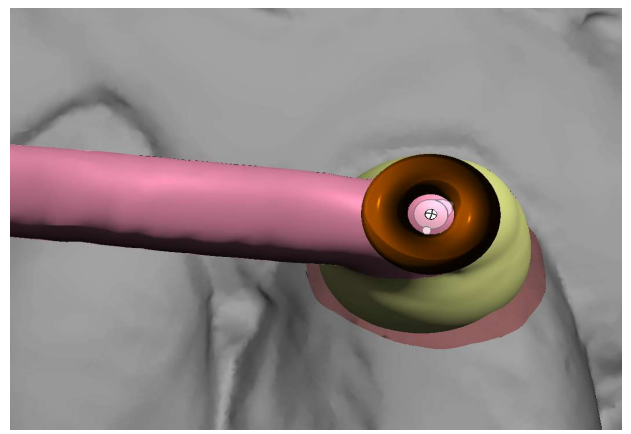

(b)

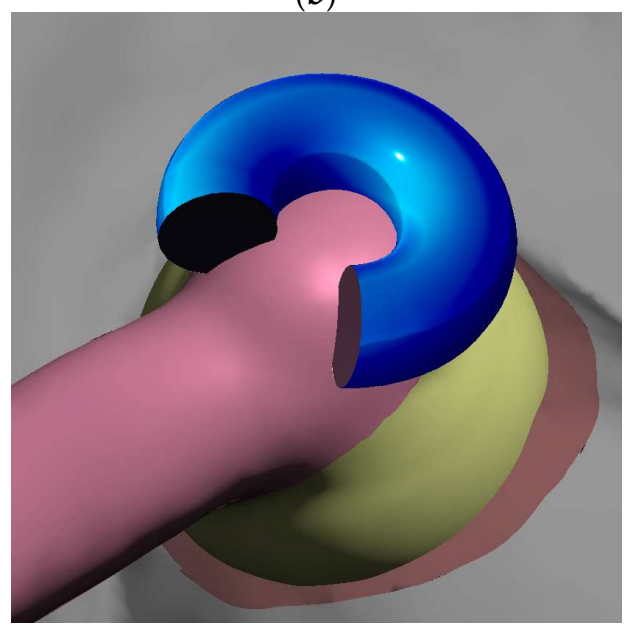

(d)

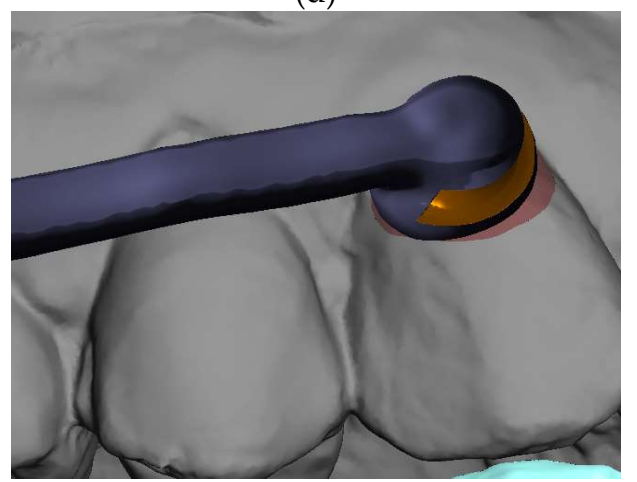

(f)

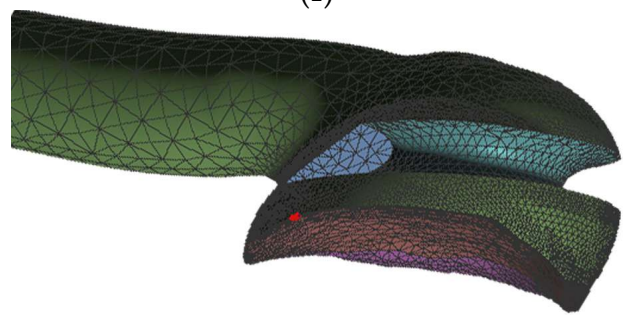

(h)

Figure 11. creation of feature supporting anchorage of the elastic bands. (a) Step in connecting area between distalizer's arm and the canine base is smoothed - different from Figure 9c. The purpose is better comfort and resiliency during chewing.; (b) Import of a primitive donut shape from basic Meshmixer gallery allows faster subtraction of desired shape for loaded elastic band; (c) Quadrant not necessary for elastic retention shall be preserved to increase the strength of this part of distalizer; (d) Reshaping of the reduced donut intended for Boolean subtraction (Select both objects and apply Boolean Difference in precise Solution mode, preferably use Intersection curves otherwise default setting in Meshmixer) Quadrant not necessary for elastic retention shall be preserved to increase the 
strength of this part of distalizer; (e) Before subtraction position properly so the long axis of future loaded elastic is pointing towards the lower anchor point - button or precision cut; (f) Result of Boolean difference subtraction of adapted donut shape; (g) Result of Boolean difference between intraoral scan and the canine part of the distalizer - base of the canine pad is now fully individualized; (h) profile view of the mesial end of distalizer with groove intended for elastic band;

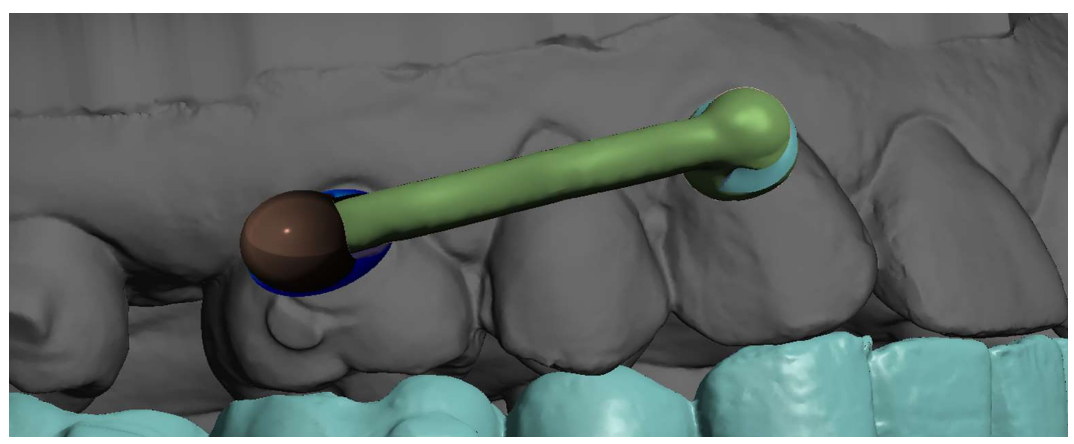

(a)

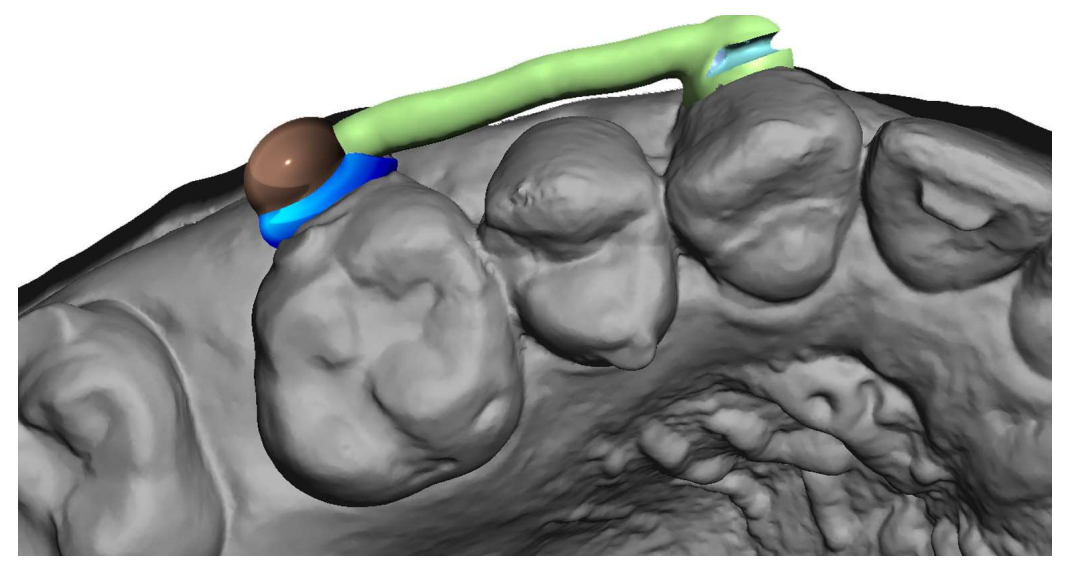

(b)

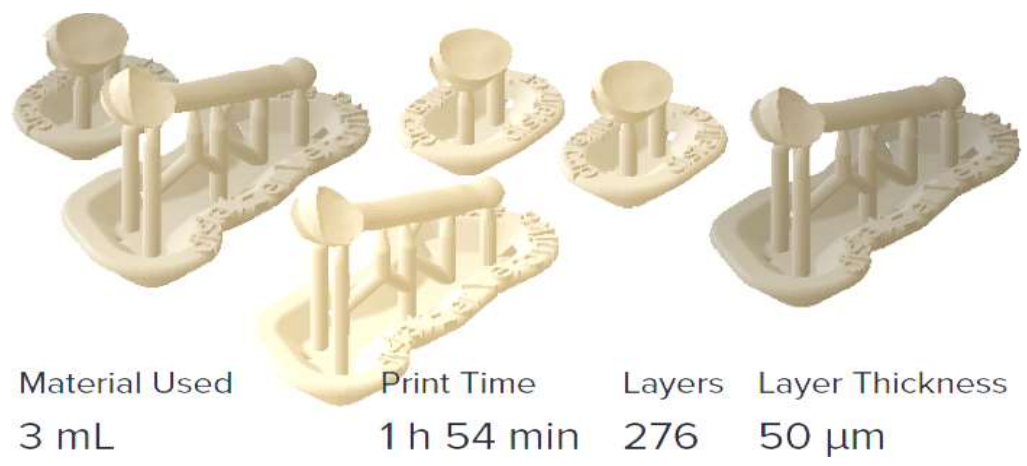

(c)

Figure 12. Final distalizer before 3D printing; (a) lateral view; (b) apical view; (c) 3D printing set-up for $50 \mu$ printing resolution with visible supports, always 3 clones were printed for possible servicing reasons, material Denture teeth, shade A2.

2.5 Method of 3D printing and post-processing

$3 \mathrm{D}$ printing and post-processing protocols are technically described in chapter 2.2 Here we explain more practical aspects of post-prints handling with their visual explanations. Figure 13. shows 3D printed example of distalizer designed ion the previous chapter. The material Denture teeth Resin Formlabs/Dentca [60,61, 63] requires washing in IPA only 10 minutes (Figure 13a, b). We have used sonic IPA bath for 10 minutes. Second 
step is post-curing of the prints, for this material is quite specific. In our protocol we have followed the official guidelines $[63,64]$ and we have used a glass container with glycerin. After preheating the glycerin to $80^{\circ} \mathrm{C}$ in Form Cure, we have used heat resistant own gear to keep the distalizers fully submerged in the glycerin during post-curing. We kept the container inside Form Cure for 30 minutes as prescribed. After the first 30-minute postcure, we have flipped the small distalizers to the opposite side and post-cured them again for another 30 minutes (Figure 13c). It is important to remember, that the glycerin during this process will get very hot $\left(80^{\circ} \mathrm{C}\right)$ and heat resistant silicone tongs shall be used to insert and remove the distalizers from the solution.

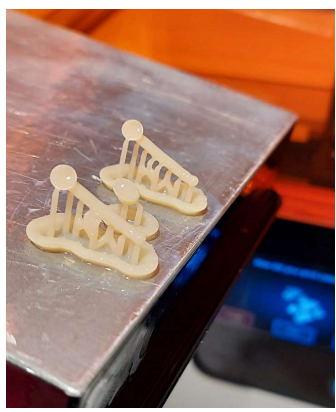

(a)

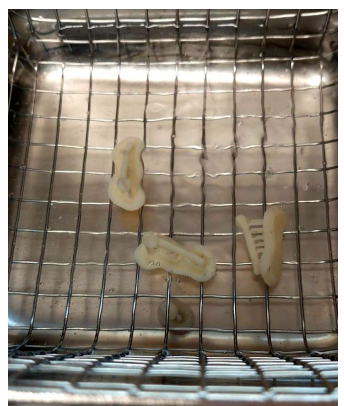

(b)

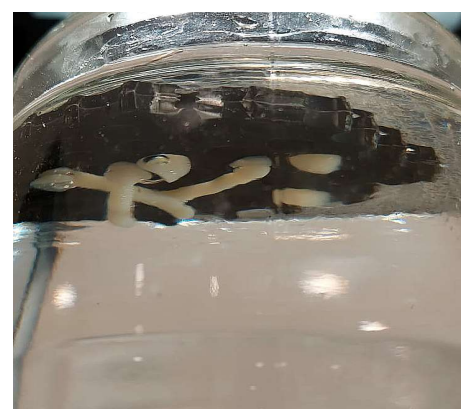

(c)

Figure 13. (a) Fresh 3D printed 3 clones of a distalizer from Denture teeth resin material; (b) Sonic IPA bath for 10 minutes; (c) Post-curing submerged in glycerin in $80^{\circ} \mathrm{C}$.

Figure 14 shows high-contrast zoomed view of post-cured distalizers from Denture Teeth resin material in shade A2. Surface shows remnants of the supports, these can be polished by doctor during clinical application if necessary. Two of the clones are a spare.

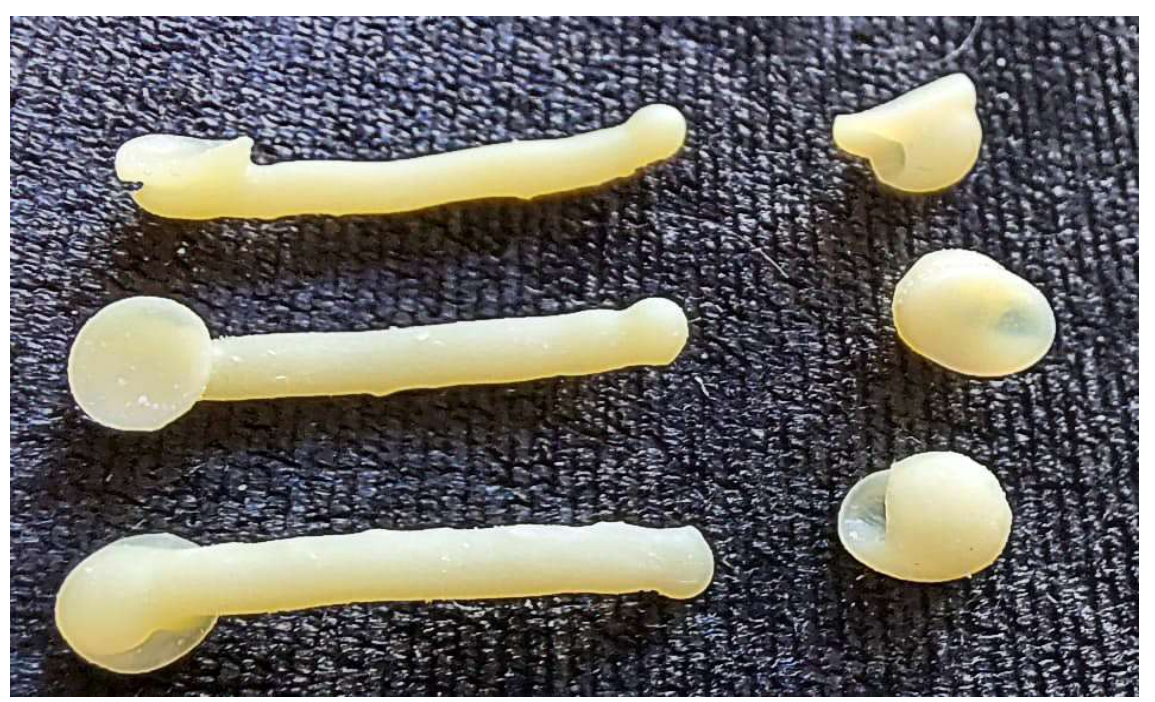

Figure 14. Three clones of 3D printed distalizer from material Denture Teeth, shade A2 


\subsection{Method of clinical application and materials}

Clinical application takes advantage from the individualized base on each terminal pad of the distalizer. The surface of such individualized base exactly matches the tooth surface in pre-planned particular position and orientation. Figure 15 describes the sequence of trying out, sandblasting, etching and final bonding of the distalizer.

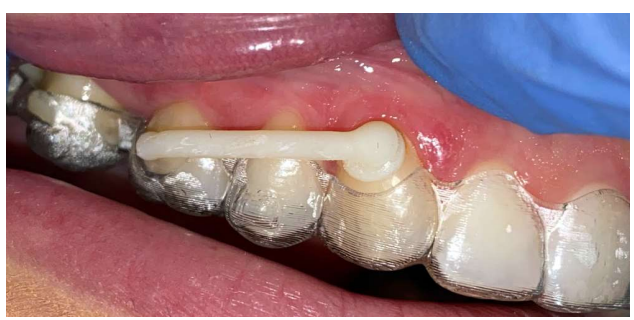

(a)

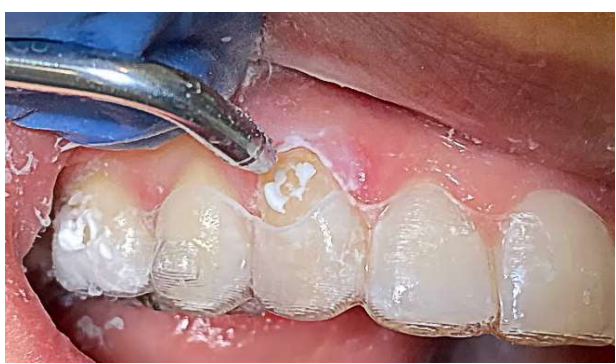

(c)

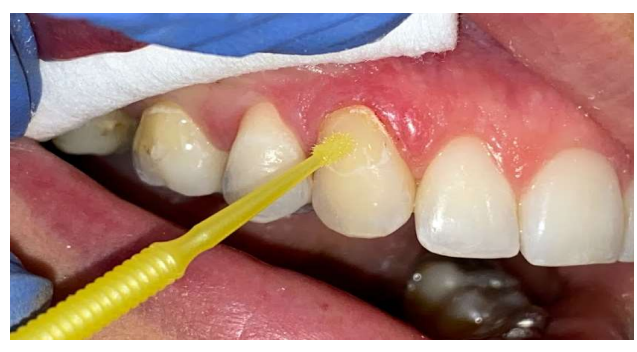

(e)

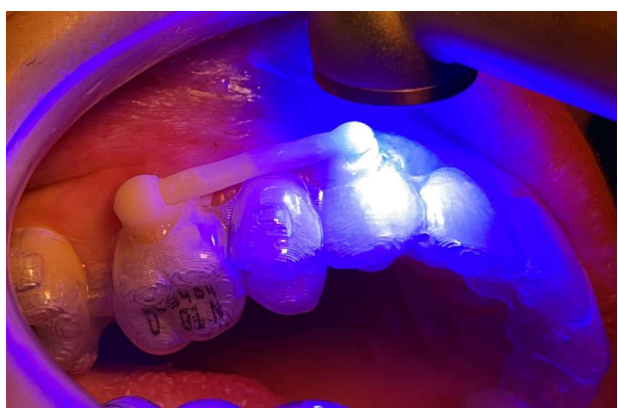

(g)

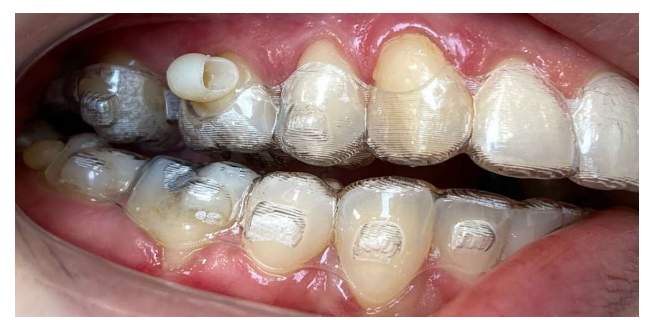

(b)

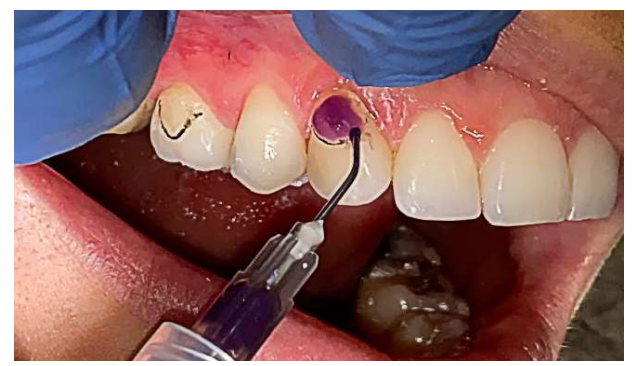

(d)

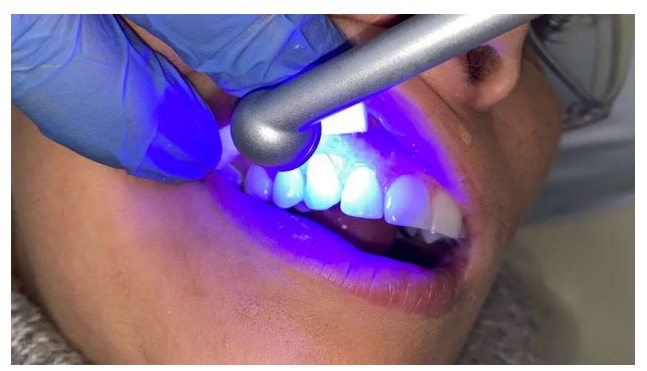

$(\mathbf{f})$

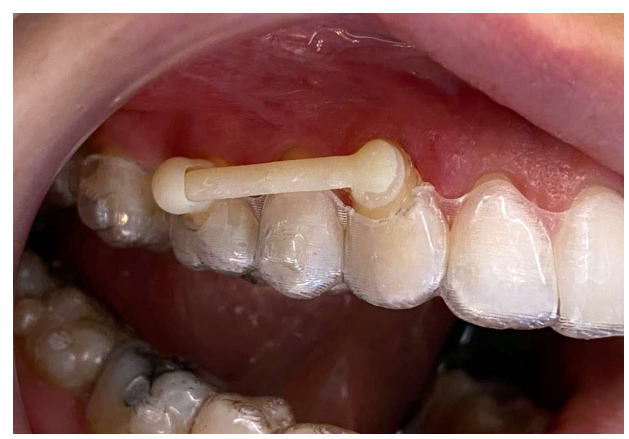

(h)

Figure 15. Clinical application of personalized 3D printed distalizer in combination with CAT (Invisalign ${ }^{\circledR)}$; (a) Initial „try-out"of the mesial part of distalizer with its „arm“; (b) „try-out"of the distal smaller part of distalizer; (c) Circular sandblasting $5 \mathrm{~s}$ each of the surface, this is proven to significantly increase bond adhesion strength [67]; (d) standard enamel etching with 37,5\% phosphoric acid, rinsing and drying; (e) application of $7^{\text {th }}$ generation Single Bond Universal from ESPE; (f) Standard Light curing of the bond; (g) Standard Light curing for final adhesion od 
distalizer with micro layer of $3 \mathrm{M}^{\mathrm{TM}}$ Transbond ${ }^{\mathrm{TM}}$ PLUS Color Change Adhesive; (h) Final appearance of the distalizer before activation;

The last step before distalizer activation is to remove possible excessive remnants of the adhesive with the dental bur softer than enamel and harder than the polymerized Transbond ${ }^{\mathrm{TM}}$ glue. Removal of upper aligner is followed by cleaning procedure (Figure 16). The planned gap for the adhesive between tooth surfaces and the distalizer is usually 50 microns.

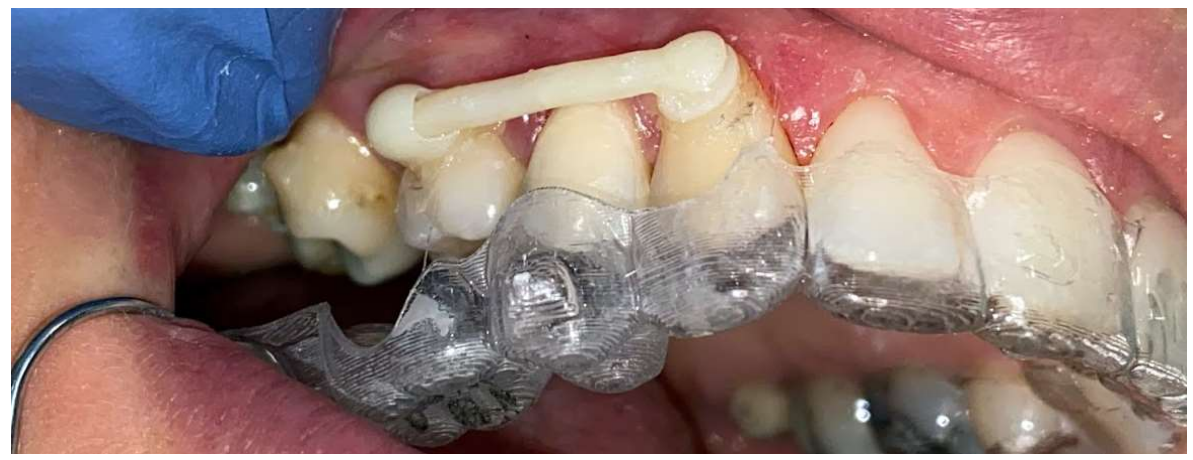

Figure 16. Fixed distalizer in hybrid treatment with CAT (Invisalign $\left.{ }^{\circledR}\right)$; before activation.

The final step is activation of the distalizer which means that the forces from the elastics will be applied. The activation is obtained by the use of two types of elastics: the first month 0.25 inch $(6,35 \mathrm{~mm}), 6 \mathrm{oz}(170 \mathrm{~g})$; and from the second month 0.19 inch $(4,8 \mathrm{~mm}), 8$ oz $(227 \mathrm{~g})$, to be used from the second month of treatment. Figure 17 shows the distalizer with elastic band from the canine pad to the button on tooth 47.

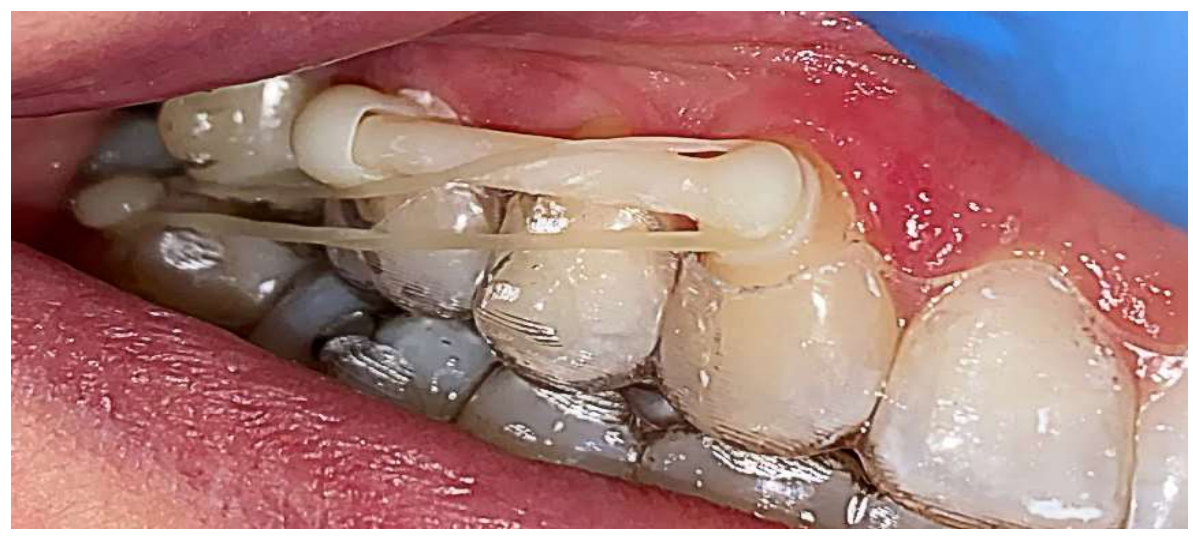

Figure 17. Fixed distalizer in hybrid treatment with CAT (Invisalign $\left.{ }^{\circledR}\right)$; after activation.

\section{Results}

The main goal of this paper was to introduce and practically accomplish the novel approach of 3D printed distalizer in hybrid orthodontic treatment. This has been achieved and is documented in procedure description in the previous chapter.

The secondary objective was to evaluate if any of the tested photopolymers is inappropriate for this intention with showing significantly more frequent debonding or breakage. Table 5 shows this evaluation of the set of 12 complete treatments with described method, where two groups of 6 patients were treated with the distalizer from one of the observed biocompatible photopolymers. To complete statistically more useful - larger set of treatments under such strict election criteria, it would require many more years. Only standard testing of the significance of the difference is feasible. The interpretation of the test results would be statistically better on a larger dataset; however, the time necessary 
to for such extensive treatment in combination of clinical selection criteria (unilateral distalization) would require many more years of observation. As the pace of development of advanced photopolymers in dental 3D printing domain is quick, years-long research would most probably bring information on already obsolete and discontinued dental materials.

Table 5. Results of clinical evaluation for secondary objective

\begin{tabular}{cccccccc}
\hline ID & Sex & Age & $\begin{array}{c}\text { Distalization Time in weeks un- } \\
\text { target }\end{array}$ & $\begin{array}{c}\text { total } \\
\text { til target achieved } \\
\text { failiures }{ }^{1}\end{array}$ & $\begin{array}{c}\text { material } \\
\text { patient had }\end{array}$ & $\begin{array}{c}\text { what material pa- } \\
\text { tient would prefer } \\
\text { next time }\end{array}$ \\
\hline$\#$ & {$[\mathrm{M} / \mathrm{F}]$} & {$[$ years] } & {$[\mathrm{mm}]$} & {$[$ weeks] } & $\#$ & [CLEAR/WHITE] [CLEAR/WHITE] \\
\hline 1 & $\mathrm{~F}$ & 25 & 2.50 & 19 & 2 & CLEAR & CLEAR \\
2 & $\mathrm{~F}$ & 26 & 3.00 & 22 & 2 & WHITE & WHITE \\
3 & $\mathrm{~F}$ & 31 & 3.50 & 20 & 0 & WHITE & CLEAR \\
4 & $\mathrm{M}$ & 23 & 3.00 & 24 & 0 & CLEAR & WHITE \\
5 & $\mathrm{M}$ & 29 & 4.50 & 33 & 1 & CLEAR & CLEAR \\
6 & $\mathrm{~F}$ & 30 & 4.00 & 32 & 0 & CLEAR & CLEAR \\
7 & $\mathrm{~F}$ & 31 & 2.50 & 21 & 0 & WHITE & CLEAR \\
8 & $\mathrm{~F}$ & 37 & 3.00 & 25 & 0 & CLEAR & CLEAR \\
9 & $\mathrm{M}$ & 28 & 3.50 & 26 & 0 & WHITE & CLEAR \\
10 & $\mathrm{~F}$ & 20 & 5.00 & 30 & 1 & WHITE & CLEAR \\
11 & $\mathrm{~F}$ & 21 & 5.50 & 36 & 1 & CLEAR & CLEAR \\
12 & $\mathrm{~F}$ & 26 & 3.00 & 20 & 0 & WHITE & CLEAR \\
\hline
\end{tabular}

${ }^{1}$ Total sum of debonding and breaking.

The results of the classic testing showed no significant findings due to the low numbers. Results are shown in the summary Table 6. There is a combination of descriptive statistics and testing (only those P values without an asterisk are "outcomes"). 
Table 6. Patients characteristics and bivariate analysis

\begin{tabular}{|c|c|c|c|c|c|}
\hline \multirow[t]{2}{*}{ Variable } & \multirow{2}{*}{$\begin{array}{l}\text { Statistics or cate- } \\
\text { gory }\end{array}$} & \multicolumn{2}{|c|}{ Group } & \multirow[t]{2}{*}{ P value } & \multirow[t]{2}{*}{ Statistical test } \\
\hline & & WHITE & CLEAR & & \\
\hline \multirow{3}{*}{ Age [years] } & $\mathrm{n}$ & 6 & 6 & n.a. & \\
\hline & mean \pm SD & $27.0 \pm 4.10$ & $27.5 \pm 5.79$ & \multirow{2}{*}{$0.866^{*}$} & \multirow{2}{*}{ t-test } \\
\hline & median (range) & $27(20-31)$ & $27(21-37)$ & & \\
\hline \multirow[t]{2}{*}{ Sex } & male & 1 & 2 & \multirow{2}{*}{$0.591^{*}$} & \multirow{2}{*}{ Fisher exact test } \\
\hline & female & 5 & 4 & & \\
\hline \multirow[t]{2}{*}{ Side of oral cavity } & left & 3 & 3 & \multirow{2}{*}{$0.999^{*}$} & \multirow{2}{*}{ Fisher exact test } \\
\hline & right & 3 & 3 & & \\
\hline \multirow[t]{2}{*}{$\begin{array}{l}\text { Distance to distalization target } \\
{[\mathrm{mm}]}\end{array}$} & mean \pm SD & $3.42 \pm 0.861$ & $3.75 \pm 1.129$ & \multirow[t]{2}{*}{0.578} & \multirow[t]{2}{*}{ t-test } \\
\hline & median (range) & $3.25(2.5-5.0)$ & $3.5(2.5-5.5)$ & & \\
\hline \multirow[t]{2}{*}{$\begin{array}{l}\text { Time until target achieved } \\
\text { [weeks] }\end{array}$} & mean \pm SD & $23.2 \pm 4.01$ & $28.2 \pm 6.49$ & \multirow[t]{2}{*}{0.223} & \multirow[t]{2}{*}{ Mann-Whitney test } \\
\hline & median (range) & $21.5(20-30)$ & $28.5(19-36)$ & & \\
\hline Total failures ${ }^{a}$ & mean $^{\mathrm{b}}$ (Poisson CI) & $0.5(0.10-1.46)$ & $0.67(0.18-1.71)$ & 0.706 & Poisson regression \\
\hline \multirow[t]{2}{*}{ Material preference } & white & 1 & 1 & \multirow{2}{*}{0.999} & \multirow{2}{*}{ Fisher exact test } \\
\hline & clear & 6 & 6 & & \\
\hline \multicolumn{6}{|c|}{ Abbreviations: SD - standard deviation, CI - confidence interval } \\
\hline \multicolumn{6}{|c|}{${ }^{*}$ For descriptive (not inferential) purpose only } \\
\hline \multicolumn{6}{|c|}{ a Total sum of debonding and breaking } \\
\hline $\mathrm{b}$ Expected number of events & & & & & \\
\hline
\end{tabular}

The results on debonding characteristics and their distribution in patients show 7 failures in total. Characteristics are:

- Total 7 failures consisted from 4 debonding incidents and 3 breaks of material

- Only in two patients happened double failure (one debonding and one breaking)

- Both patients with double-failures preferred the same material in the final question

- Another three failures were separate incidents in separate patients

Figure 18 shows extends of distalization for both groups. The range of data shown in Figure 18 may be viewed as an estimate of the range of possible outcomes, and the difference between two mean data points on the graph as an estimate of the expected difference between these two materials (the estimates were made using bivariate analysis, i.e., without taking into account the influence of other factors on the outcomes). Medians are depicted as lines within the boxes and the respective mean with a diamond sign. The "whiskers" extend from the ends of the box to the minimum and maximum data values. The circle denotes an observation whose value is distant from the rest of the sample. Considering the range of clinically observed values this data point is not a genuine outlier, rather it is a consequence of the small number of observations. 


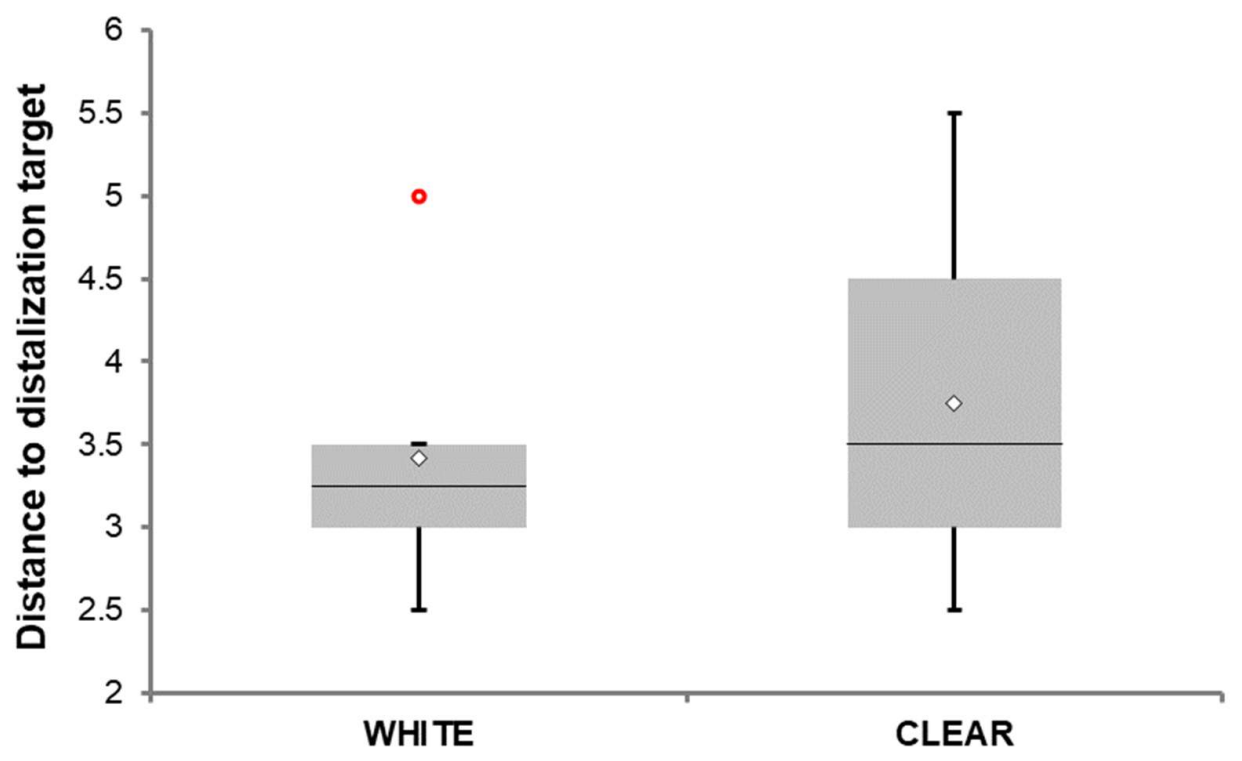

Figure 18. Data distribution of distance to distalization target stratified by type of photopolymer. The difference between group means was not statistically significant $(P=0.578)$.

Finally, our tertiary objective was to examine patients' preference after they had completed treatment with either white or clear (transparent) material. Only two patients out of a total 12 patients (16.7\%) preferred white resin over the transparent one. The question was "Which of these two materials would you consider more aesthetically appeasing for a distalizer if you can choose?". They were shown examples of both materials together.

Finally, our final tertiary objective was to examine patients' preference after they had completed treatment with either white or clear (transparent) material. Only two patients out of a total 12 patients (16.7\%) preferred white resin over the transparent one. Since the proportions of patients preferring transparent material were the same with a maximum difference by $3.2 \%$ assessed by the binomial test for independent proportions (approximate Miettinen $95 \%$ confidence interval $=-0.4679$ to 0.4679 ), we can conclude that the preferences were comparable in the study arms. In other words, our findings suggest that patients have preferred transparent material independently of the material used for treatment.

\section{Discussion}

This paper introduces personalized distalizer treatment of a Class II malocclusion in adults. Current evidence suggests that orthodontic treatment of such malocclusion should generally be provided during adolescence. However, early treatment of Class II has been advocated to reduce the risk of incisal trauma for example use of Forsus Fatigue Resistant Device as a non-compliance mandibular advancer after rapid palatal expansion was found to be effective at reducing the sagittal jaw discrepancy, successful at controlling the patient unfavourable growth pattern, and beneficial for the child aesthetics Goracci, Cacciatore 2017 [68]. This paper proves that non-compliance orthodontic devices are more achievable with personalized $3 \mathrm{D}$ printing.

This paper introduces a novel method of personalized orthodontic treatment with 3D printed distalizer from photopolymer resin. This has been never published before. Closest research in this topic is research published in journal of clinical orthodontics in 2020 by Simon Graf on account of CAD/CAM metallic printing of skeletally anchored upper molar distalizer [69]. This research is about skeletally borne metallic palatal distalizer. This paper is about photo-polymeric $3 \mathrm{D}$ printed tooth borne vestibular distalizer. 
Authors of this paper have recently published research focused on 3D printed orthodontic auxiliary - power-arm [70] using Finite Element Analysis approach for 3D design. Similar research, also implementing the Finite Element Method to evaluate the tooth movement efficacy of retraction springs made of a new $\beta$-titanium alloy was published with Tamaya et al. [71]. The Finite Element Method with combination of advanced Artificial Intelligence features like 3D Convolution Neural Networks has bright perspective in future of individualized approach for 3D design of intraoral appliances and thus effective orthodontic treatment [72].

Results showed no significant clinical difference between both tested materials. Despite white photopolymer was 3D printed on better resolution (50 microns versus 100microns), there was no clinical impact. Debonding incidents were mostly caused by patientadmitted non-compliance during eating and material damage was rare. Interesting aspect of this study is that all orthodontic patients 'intraoral situation was monitored remotely with tele-Health solution - Dental Monitoring, which is judged positively with orthodontists Dalessandri et al. 2021 [73] Small differences in material properties had no clinically relevant impact on the distalizers performance. Implication of these findings might suggest wider possibility of suitable materials - dedicated dental resins. In 3D modelling of individualized distalizers we recommend to use Open-Source free software like MeshMixer or Blender. Also, for creation of other orthodontic devices confirmed with recent publication of Canova et al. 2021 these are preferred with younger clinicians with more extensive digital skills [74].

3D printing in orthodontics is now greatly focused albeit not limited to orthodontic aligners and recent research of Zinelis et al. 2021 shows that mechanical properties are depending not only on type of resin but also on method of 3D printing affecting their clinical efficacy [75]

The results can be interpreted from the perspective of previous studies mentioned in introduction that photo-polymeric dental resins can be used for 3D printing of individualized distalizer with the comparable clinical efficiency. From the perspective of extraordinary personalization of intraoral appliances with biocompatible 3D printing this approach has high potential also in addressing issues linked with cleft therapy with dynamically changing intraoral morphology [76].

The general findings of this paper are, that 3D printed biocompatible personalized distalizers can be successfully applied in tooth-borne hybrid treatment in class II unilateral malocclusions. Results of comparison of two biocompatible photopolymers showed that small differences in their material properties are not crucial to their clinical performance. Implications resulting from these finding could advocate future wide use of these materials as the dental resins gain better properties and thus intraoral resiliency [77, 78].

This research showed preference of patients towards transparent biocompatible material instead of white A2 shade. As the final tertiary objective was to evaluate patient preference of material. Surprisingly only one patient who was treated with clear distalizer preferred white appliance "for the next time". And only one of the patients wearing white shade A2 distalizers preferred white distalizer again, the rest would prefer transparent one if possible. Despite it seems obvious that transparent material for distalizers was aesthetically more pleasing, the limitation of this consideration is that patients were confronted only with one particular dental shade (A2).

Another limitation of this study is a small set of clinical treatments observed. To widen the set to gather statistically useful numbers of clinical application it would be necessary to either:

1. reduce strictness of clinical conditions like I. skeletal class patients or bilateral distalization situations

2. or prolong the research for more years.

As these biocompatible dental resins are continuously updated prolonged research would bring results that would be already obsolete. 
A future contribution of this paper might be relying in the realization of orthodontists, that individualization of the orthodontic treatment might be the key for efficient treatment as it was in the past. That the incoming smart materials for 3D printing might return the treatment back to their hands. Orthodontics is an emerging field in which polymers have attracted the enormous attention of researchers. In particular, thermoplastic materials have a great future utility in orthodontics, both as aligners and as retainer appliances. The most promising polymeric smart materials are also discussed for their relevance to future orthodontic applications.

\section{Conclusions}

3D printed biocompatible individualized distalizer from photo-polymeric resin, can be used for tooth-borne hybrid approach in class II unilateral malocclusions treatment.

Upon evaluation of frequency of debonding of the distalizers on the set of 12 complete orthodontic treatments, we can conclude that there was no significant difference between materials regarding this measure.

Patient evaluation of aesthetically most pleasing material for distalizer was selected the clear(transparent) material to an alternative white (A2) material.

Author Contributions: Conceptualization, A.T. and I.V.; methodology, A.T., I.W., ; software, A.T. I.W.; validation, A.T., B. N., I.W.; formal analysis, A.T., I.W.; investigation, A.T.; resources, A.T.; data curation, A.T., I.W,; writing-original draft preparation, A.T.; writing-review and editing, A.T., I.W., B.N., W.U.; visualization, A.T.; supervision, I.V.; project administration, A.T.; funding acquisition, I.V.; All authors have read and agreed to the published version of the manuscript."

Funding: This research was funded by the KEGA grant agency of the Ministry of Education, Science, Research, and Sport of the Slovak Republic (Grant No. 081UK-4/2021); Data analysis was supported by the grants APVV: SK-BY-RD-19-0019 and KEGA: 041UK$4 / 2020$.

Institutional Review Board Statement: The study was conducted according to the guidelines of the Declaration of Helsinki, and no approval was necessary by the Ethics Committee. Ethical review and approval were waived for this study, due to the fact that no experimental materials or approaches were used. All used materials and machines were fully certified and are still available on the market. Dental LT Clear is tested at NAMSA, Chasse sur Rhône in France, and is certified biocompatible per EN-ISO 109931:2009/AC:2010. The biocompatible resin material follows ISO Standards: EEN ISO 1641:2009, EN-ISO 10993-1:2009/AC:2010, EN-ISO 10993-3:2009, EN-ISO 10993-5:2009, EN 908:2008.

3D printer - Formlabs Form 2 was the first and only stereolithography 3D printer to be validated by Materialise as part of the Materialize first FDA-cleared process to create accurate patient-specific anatomical models for diagnostic use. Formlabs electrical equipment is manufactured in facilities with the following QMS certifications. Form 2(Quality system standards) ISO 9001:2015 and ISO 14001:2015.

Formlabs, Inc., located in Somerville, Massachusetts, USA, certifies that: Formlabs Form 2 3D printers are assembled within the European Union in Hungary and Formlabs Dental SG Resin and LT Clear Resin are manufactured within the European Union in The Netherlands.

Informed Consent Statement: Written informed consent was obtained from all subjects involved in the study.

Data Availability Statement: We fully adhere to Data Availability Statements in section "MDPI Research Data Policies" at https://www.mdpi.com/ethics. 
Acknowledgments: We acknowledge technological support of digital Dental lab infrastructure of 3Dent Medical s.r.o company as well as dental clinic Sangre Azul s.r.o.

Conflicts of Interest: The authors declare no conflict of interest.

\section{Patents}

This section is not mandatory but may be added if there are patents resulting from the work reported in this manuscript.

\section{References}

1. Huang, H.-M. Medical Application of Polymer-Based Composites. Polymers 2020, 12, 2560. https://doi.org/10.3390/polym12112560

2. Scribante, A.; Vallittu, P.K.; Özcan, M.; "Fiber-Reinforced Composites for Dental Applications", BioMed Research International, vol. 2018, Article ID 4734986, 2 pages, 2018. https://doi.org/10.1155/2018/4734986

3. Burgard, N.; Kienitz, M.; Jourdan, C.; Rüttermann, S. The Influence of Modified Experimental Dental Resin Composites on the Initial In Situ Biofilm-A Triple-Blinded, Randomized, Controlled Split-Mouth Trial. Polymers 2021, $13,2814$. https://doi.org/10.3390/polym13162814

4. Grzebieluch, W.; Kowalewski, P.; Grygier, D.; Rutkowska-Gorczyca, M.; Kozakiewicz, M.; Jurczyszyn, K. Printable and Machinable Dental Restorative Composites for CAD/CAM Application-Comparison of Mechanical Properties, Fractographic, Texture and Fractal Dimension Analysis. Materials 2021, 14, 4919. https://doi.org/10.3390/ma14174919

5. Chung, Y.-J.; Park, J.-M.; Kim, T.-H.; Ahn, J.-S.; Cha, H.-S.; Lee, J.-H. 3D Printing of Resin Material for Denture Artificial Teeth: Chipping and Indirect Tensile Fracture Resistance. Materials 2018, 11, 1798. https://doi.org/10.3390/ma11101798

6. Sfondrini, M.F.; Vallittu, P.K.; Lassila, L.V.J.; Viola, A.; Gandini, P.; Scribante, A. Glass Fiber Reinforced Composite Orthodontic Retainer: In Vitro Effect of Tooth Brushing on the Surface Wear and Mechanical Properties. Materials 2020, $13,1028$. https://doi.org/10.3390/ma13051028

7. Chen, S.; Yang, J.; Jia, Y.-G.; Lu, B.; Ren, L. A Study of 3D-Printable Reinforced Composite Resin: PMMA Modified with Silver Nanoparticles Loaded Cellulose Nanocrystal. Materials 2018, 11, 2444. https://doi.org/10.3390/ma11122444

8. Tappa, K.; Jammalamadaka, U. Novel Biomaterials Used in Medical 3D Printing Techniques. J. Funct. Biomater. 2018,9 , 17. https://doi.org/10.3390/jfb9010017

9. Melo, M.A. Bacterial Interactions with Dental and Medical Materials. J. Funct. Biomater. 2020, 11, 83. https://doi.org/10.3390/jfb11040083

10. Mai, H.-N.; Hyun, D.C.; Park, J.H.; Kim, D.-Y.; Lee, S.M.; Lee, D.-H. Antibacterial Drug-Release Polydimethylsiloxane Coating for 3D-Printing Dental Polymer: Surface Alterations and Antimicrobial Effects. Pharmaceuticals 2020, 13, 304. https://doi.org/10.3390/ph13100304

11. Jindal, P., Juneja, M., Bajaj, D., Siena, F.L. and Breedon, P. (2020), "Effects of post-curing conditions on mechanical properties of 3D printed clear dental aligners", Rapid Prototyping Journal, Vol. 26 No. 8, pp. 1337-1344. https://doi.org/10.1108/RPJ-04-20190118

12. Vasques, M.T.; Mori, M.; Laganá, D.C.; Three-dimensional printing of occlusal devices for temporomandibular disorders by using a free CAD software program: A technical report, The Journal of Prosthetic Dentistry, Volume 123, Issue 2, 2020, Pages 232-235, ISSN 0022-3913, https://doi.org/10.1016/j.prosdent.2018.12.017.

13. Milovanović, A.; Sedmak, A.; Golubović, Z.; Mihajlović, K.Z.; Žurkić,A.; Trajković, I.; Milošević, M.; The effect of time on mechanical properties of biocompatible photopolymer resins used for fabrication of clear dental aligners, Journal of the Mechanical Behavior of Biomedical Materials, Volume 119, 2021, ISSN 1751-6161, https://doi.org/10.1016/j.jmbbm.2021.104494.

14. Scribante, A.; Gallo, S.; Turcato, B.; Trovati, F.; Gandini, P.; Sfondrini, M.F. Fear of the Relapse: Effect of Composite Type on Adhesion Efficacy of Upper and Lower Orthodontic Fixed Retainers: In Vitro Investigation and Randomized Clinical Trial. Polymers 2020, 12, 963. https://doi.org/10.3390/polym12040963

15. Sfondrini, M.F.; Gallo, S.; Turcato, B.; Montasser, M.A.; Albelasy, N.F.; Vallittu, P.K.; Gandini, P.; Scribante, A. Universal Adhesive for Fixed Retainer Bonding: In Vitro Evaluation and Randomized Clinical Trial. Materials 2021, 14, 1341. https://doi.org/10.3390/ma14061341

16. Proffit, W.R.; Fields, H. W.; Moray, L.J.; Prevalence of malocclusion and orthodontic treatment need in the United States: estimates from the NHANES III survey. The International Journal of Adult Orthodontics and Orthognathic Surgery. 1998 ;13(2):97106. PMID: 9743642.

17. Ke, Y., Zhu, Y. \& Zhu, M. A comparison of treatment effectiveness between clear aligner and fixed appliance therapies. BMC Oral Health 19, 24 (2019). https://doi.org/10.1186/s12903-018-0695-z

18. Rossini G, Parrini S, Castroflorio T, Deregibus A, Debernardi C.L. Efficacy of clear aligners in controlling orthodontic tooth move-ment: A systematic review. Angle Orthod 1 September 2015; 85 (5): 881-889. doi: https://doi.org/10.2319/061614-436.1 
19. Nanda, R.; Garino, F.; Ojima, K.; Castroflorio, T.; Parrini, S.; Chapter 12: The hybrid approach in class II malocclusions treatment. In Principles and Biomechanics of Aligner Treatment - E-Book, 1st ed.; Publisher: Elsevier Health Sciences, 304 pages, Country, 2021; Volume 1, pp. 149-160. ISBN 9780323683838

20. Bolla E, Muratore F, Carano A, et al. Evaluation of maxillary molar distalization with the distal jet a comparison with other contemporary methods Angle Orthod 2002;72: 481-494.

21. Grec RH, Janson G, Branco NC, et al. Intraoral distalizer effects with conventional and skeletal anchorage a meta-analysis Am J Orthod Dentofacial Orthop 2013;143: 602-615.

22. Ravera S, Castroflorio T, Garino F, et al. Maxillary molar distalization with aligners in adult patients a multicenter retrospective study Prog Orthod 2016;17: 12.

23. Barakat D, Bakdach WM, Youssef M. Treatment effects of Carriere Motion Appliance on patients with class II malocclusion: A systematic review and meta-analysis. International Orthodontics. 2021 Jun 12. 19. 10.1016/j.ortho.2021.05.005.

24. Scribante, A.; Gallo, S.; Bertino, K.; Meles, S.; Gandini, P.; Sfondrini, M.F. The Effect of Chairside Verbal Instructions Matched with Instagram Social Media on Oral Hygiene of Young Orthodontic Patients: A Randomized Clinical Trial. Appl. Sci. 2021, 11, 706. https://doi.org/10.3390/app11020706

25. Rossini G, Parrini S, Deregibus A, et al. Controlling orthodontic tooth movement with clear aligners. An updated systematic review regarding efficacy and efficiency J Aligner Orthod 2017;1: 7-20.

26. Garino F, Castroflorio T, Daher S, et al. Effectiveness of composite attachments in controlling upper-molar movement with aligners J Clin Orthod 6, 2016;50: 341-347.

27. Richter DD, Nanda RS, Sinha PK, et al. Effect of behavior modification on patient compliance in orthodontics Angle Orthod 1998;68: 123-132.

28. Lombardo L, Colonna A, Carlucci A, et al. Class II subdivision correction with clear aligners using intermaxilary elastics Prog Orthod 2018;1: 19-32.

29. Nedwed V \& Miethke RR. Motivation, acceptance and problems of invisalign patients J Orofac Orthop 2005;66: 162-173.

30. Rosvall MD, Fields HW, Ziuchkovski J, et al. Attractiveness, acceptability, and value of orthodontic appliances Am J Orthod Dentofacial Orthop 2009;135: 276.e1-276.e12.

31. Shah N. Compliance with removable orthodontic appliances Evid Based Dent 2017;18: 105-106.

32. Carano A \& Testa M. The distal jet for upper molar distalization J Clin Orthod 1996;30: 374-380.

33. Antonarakis GS \& Kiliaridis S. Maxillary molar distalization with noncompliance intramaxillary appliances in class II malocclusion a systematic review Angle Orthod 2008;78: 1133-1140.

34. Carano A, Testa M \& Siciliani G. The lingual distalizer system Eur J Orthod 1996;18: 445-448.

35. Hilgers JJ. The pendulum appliance for class II noncompliance therapy J Clin Orthod 1992;26: 706-714.

36. Marure PS, Patil RU, Reddy S, et al. The effectiveness of pendulum, K-loop, and distal jet distalization techniques in growing children and its effects on anchor unit a comparative study J Indian Soc Pedod Prev Dent 2016;34: 331-340.

37. Byloff FK, Darendeliler MA, Clar E, et al. Distal molar movement using the pendulum appliance. Part 2 the effects of maxillary molar root uprighting bends Angle Orthod 1997;67: 261-270.

38. Chaqués-Asensi J \& Kalra V. Effects of the pendulum appliance on the dentofacial complex J Clin Orthod 2001;35: 254-257.

39. Byloff FK \& Darendeliler MA. Distal molar movement using the pendulum appliance. Part 1 clinical and radiological evaluation Angle Orthod 1997;67: 249-260.

40. Ghosh J \& Nanda RS. Evaluation of an intraoral maxillary molar distalization technique Am J Orthod Dentofacial Orthop 1996;110: 639-646.

41. Carrière L. A new class II distalizer J Clinic Orthod 2004;38: 224-231.

42. Martel D. The Carriere distalizer simple and efficient Int J Orthod Milwaukee 2, 2012;23: 63-66.

43. Rodríguez HL. Unilateral application of the Carriere distalizer J Clin Orthod 3, 2011;45: 177-180.

44. Sandifer CL, English JD, Colville CD, et al. Treatment effects of the Carrière distalizer using lingual arch and full fixed appliances J World Fed Orthod 2, 2014;3: e49-e54.

45. Nanda R. Biomechanics in Clinical Orthodontics 1997; WB Saunders.

46. Yin, K., Han, E., Guo, J. et al. Evaluating the treatment effectiveness and efficiency of Carriere Distalizer: a cephalometric and study model comparison of Class II appliances. Prog Orthod. 20, 24 (2019). https://doi.org/10.1186/s40510-019-0280-2

47. Kim-Berman, H., McNamara, J. A., Jr, Lints, J. P., McMullen, C., \& Franchi, L. (2019). Treatment effects of the Carriere ${ }^{\circledR}$ Motion $3 \mathrm{D}^{\mathrm{TM}}$ appliance for the correction of Class II malocclusion in adolescents. The Angle orthodontist, 89(6), 839-846. https://doi.org/10.2319/121418-872.1

48. Khosravi R, Cohanim B, Hujoel P, et al. Management of overbite with the Invisalign appliance Am J Orthod Dentofacial Orthop 2017;151: 691-699.

49. Mantovani E, Parrini S, Coda E, et al. Micro computed tomography evaluation of Invisalign aligner thickness homogeneity Angle Orthod 2021; doi:10.2319/040820-265.1. Epub ahead of print.

50. Kinzinger GS, Wehrbein H, Gross U, et al. Molar distalization with pendulum appliances in the mixed dentition effects on the position of unerupted canines and premolars Am J Orthod Dentofacial Orthop 2006;129: 407-417.

51. Rodríguez, H.L., 2011. Unilateral application of the Carriere Distalizer. Journal of clinical orthodontics: JCO, 45(3), pp.177-180.

52. Ahmed Sh. H. Three dimensional assessment of the long-term treatment stability after maxillary first molar distalization with Carriere distalizer appliance. Life Sci J 2020;17(2):83-90]. ISSN: 1097-8135 (Print) / ISSN: 2372-613X (Online). http://www.lifesciencesite.com. 12. doi:10.7537/marslsj170220.12. 
53. Wilson, B, Nikoleta Konstantoni, Ki Beom Kim, Patrick Foley, Hiroshi Ueno; Three-dimensional cone-beam computed tomography comparison of shorty and standard Class II Carriere Motion appliance. Angle Orthod 1 July 2021; 91 (4): 423-432. doi: https://doi.org/10.2319/041320-295.1

54. Hamilton CF, Saltaji H, Preston CB, Flores-Mir C, Tabbaa S. Adolescent patients' experience with the Carriere distalizer appliance. Eur J Paediatr Dent. 2013; 14: 219- 224.

55. Areepong, D.; Kim, K.B.; Oliver, D.R.; Ueno, H.; The Class II Carriere Motion appliance: A 3D CBCT evaluation of the effects on the dentition. Angle Orthod 1 July 2020; 90 (4): 491-499. doi: https://doi.org/10.2319/080919-523.1

56. Gupta, D.K.; Tuli, A.; Jain, A.; 3D printed material application in orthodontics, Materials Today: Proceedings, Volume 28, Part 3, 2020, Pages 1635-1642, ISSN 2214-7853, https://doi.org/10.1016/j.matpr.2020.04.856.

57. Eliades T, Zinelis S. Three-dimensional printing and in-house appliance fabrication: Between innovation and stepping into the unknown. Am J Orthod Dentofacial Orthop. 2021 Jan;159(1):1-3. doi: 10.1016/j.ajodo.2020.10.012. PMID: 33357747.

58. Guarnieri FDF, Briso ALF, Ramos FdSeS, Esteves LMB, Omoto ÉM, et al. (2021) Use of auxiliary devices during retreatment of direct resin composite veneers. PLOS ONE 16(6): e0252171. https://doi.org/10.1371/journal.pone.0252171

59. Technical Data Sheet - Dental LT Clear (V2). Available online: https://formlabs-media.formlabs.com/datasheets/2001429-TDSENUS-0.pdf (accessed on 10.10.2021).

60. Formlabs Denture Resins - PKG-RS-F2-DT. Available online: https://dental.formlabs.com/store/denture-teeth-resin-11/ (accessed on 10.10.2021).

61. Form Cure time and temperature settings. Available online: https://s3.amazonaws.com/servicecloudassets.formlabs.com/media/Finishing/Post-Curing/115001414464-Form\%20Cure\%20Time\%20and\%20Temperature\%20Settings/FormCurePostCureSettings.pdf (accessed on 10.10.2021).

62. Safety Data Sheet - Dental LT Clear (V2). Available online: https://formlabs-media.formlabs.com/datasheets/2001421-SDSENEU-0.pdf (accessed on 10.10.2021).

63. Safety Data Sheet - Denture Teeth. Available online: https://formlabs-media.formlabs.com/datasheets/1902185-SDS-ENEU$0 . p d f$ (accessed on 10.10.2021).

64. Technical Data Sheet - Denture Base and Teeth. Available online: https://dental-media.formlabs.com/datasheets/1802131-TDSENEU-0.pdf (accessed on 10.10.2021).

65. Form 2-3D printer Technical Data Sheet - online: https://formlabs.com/3d-printers/form-2/ (accessed on 10.10.2021).

66. Chung, Y.-J.; Park, J.-M.; Kim, T.-H.; Ahn, J.-S.; Cha, H.-S.; Lee, J.-H. 3D Printing of Resin Material for Denture Artificial Teeth: Chipping and Indirect Tensile Fracture Resistance. Materials 2018, 11, 1798. https://doi.org/10.3390/ma11101798

67. Espinar-Escalona, E., Barrera-Mora, J.M., Llamas-Carreras, J.M. et al. Improvement in adhesion of the brackets to the tooth by sandblasting treatment. J Mater Sci: Mater Med 23, 605-611 (2012). https://doi.org/10.1007/s10856-011-4509-y

68. Goracci C, Cacciatore G. Early treatment of a severe Class II malocclusion with the Forsus fatigue resistant device. Eur J Paediatr Dent. 2017 Sep;18(3):199-207. doi: 10.23804/ejpd.2017.18.03.06. PMID: 29254343.

69. Graf S, Vasudavan S, Wilmes B. CAD/CAM Metallic Printing of a Skeletally Anchored Upper Molar Distalizer. J Clin Orthod. 2020 Mar;54(3):140-150. PMID: 32554916.

70. Thurzo, A.; Kočiš, F.; Novák, B.; Czako, L.; Varga, I. Three-Dimensional Modeling and 3D Printing of Biocompatible Orthodontic Power-Arm Design with Clinical Application. Applied Sciences 2021, 11, 9693, https://doi.org/10.3390/app11209693

71. Tamaya, N.; Kawamura, J.; Yanagi, Y. Tooth Movement Efficacy of Retraction Spring Made of a New Low Elastic Modulus Material, Gum Metal, Evaluated by the Finite Element Method. Materials 2021, 14, 2934. https://doi.org/10.3390/ma14112934

72. Thurzo, A.; Kosnáčová, H.S.; Kurilová, V.; Kosmel', S.; Beňuš, R.; Moravanský, N.; Kováč, P.; Kuracinová, K.M.; Palkovič, M.; Varga, I. Use of Advanced Artificial Intelligence in Forensic Medicine, Forensic Anthropology and Clinical Anatomy. Healthcare 2021, 9, 1545. https://doi.org/10.3390/healthcare9111545

73. Dalessandri, D.; Sangalli, L.; Tonni, I.; Laffranchi, L.; Bonetti, S.; Visconti, L.; Signoroni, A.; Paganelli, C. Attitude towards Telemonitoring in Orthodontists and Orthodontic Patients. Dent. J. 2021, 9, 47. https://doi.org/10.3390/dj9050047

74. Federici Canova, F.; Oliva, G.; Beretta, M.; Dalessandri, D. Digital (R)Evolution: Open-Source Softwares for Orthodontics. Appl. Sci. 2021, 11, 6033. https://doi.org/10.3390/app11136033

75. Spiros Zinelis; Nearchos Panayi; Georgios Polychronis; Spyridon N. Papageorgiou; Theodore Eliades. Comparative analysis of mechanical properties of orthodontic aligners produced by different contemporary 3D printers. Orthodontics \& Craniofacial Research 2021, 1 . DOI: 10.1111/ocr.12537

76. Urbanova, W.; Klimova, I.; Brudnicki, A.; Polackova, P.; Kroupova, D.; Dubovska, I.; Rachwalski, M.; Fudalej, P.S. The SlavCleft: A Three-Center Study of the Outcome of Treatment of Cleft Lip and Palate. Part 1: Craniofacial Morphology. Journal of Cranio-Maxillofacial Surgery 2016, 44, 1767-1776, doi:10.1016/J.JCMS.2016.06.010.

77. Frank Alifui-Segbaya, F.; Varma, S., Lieschke, G.J., George, R.; 3D Printing and Additive Manufacturing. Dec 2017.185191.http://doi.org/10.1089/3dp.2017.0064

78. Schuster, M., Turecek, C., Kaiser, B., Stampfl, J., Liska R.,Varga F.; Evaluation of Biocompatible Photopolymers I: Photoreactivity and Mechanical Properties of Reactive Diluents, (2007) Journal of Macromolecular Science, Part A, 44:5, 547-557, DOI: 10.1080/10601320701235958 